\title{
Systematics of the release of residual nuclei from relativistic nucleus-nucleus interactions
}

\author{
W. R. Binns, ${ }^{(a)}$ T. L. Garrard, ${ }^{(b)}$ M. H. Israel, ${ }^{(a)}$ M. P. Kertzmann, ${ }^{(c)}$ J. Klarmann, ${ }^{(a)}$ \\ E. C. Stone, ${ }^{(b)}$ and C. J. Waddington ${ }^{(c)}$ \\ ${ }^{(a)}$ Department of Physics and the McDonnell Center for the Space Sciences, Washington University, St. Louis, Missouri 63130

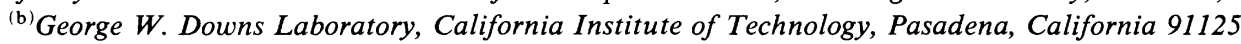

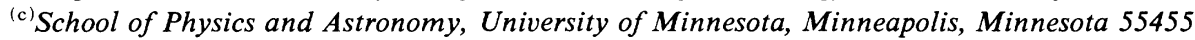

(Received 25 March 1987)

\begin{abstract}
We have examined relativistic nuclei of krypton, xenon, holmium, and gold, accelerated in a partially stripped state to a maximum rigidity of about $5.6 \mathrm{GeV}$, interacting with targets of aluminum, carbon, and polyethylene. For each projectile and target combination we determined the total and partial charge changing cross sections for the production of lighter fragments. From these measurements we have developed a new representation of the dependence of the total charge changing cross sections on beam and target charge. We have also identified simple representations of the variation of the partial cross sections with the charge of the produced fragments and shown that they are dependent on the charge and energy of the beam. The fission of gold nuclei at high energies in these various targets has also been studied.
\end{abstract}

\section{INTRODUCTION}

The interactions of relativistic heavy nuclei with target nuclei can lead to the copious release of residual fragments which have not been significantly affected in the interaction; the so called "spectator" nuclei. This paper is specifically concerned with the peripheral collisions during which these spectator nuclei are most abundantly produced. In this it differs from those studies of relativistic interactions of nuclei which are more concerned with the phenomena that occur during central collisions when the nuclei are totally disrupted. The initial objective of this study was to gain a better understanding of the passage of heavy nuclei through matter, specifically in relation to the problem of the propagation of very heavy cosmic ray nuclei through the interstellar medium and through material in and around cosmic ray detectors. In addition, we hoped to obtain a better understanding of the nuclear physics involved in these peripheral collisions. In this paper we have restricted our attention mainly to the nuclear physics aspects of the experimental results and deferred discussion of their application to the problem of cosmic ray propagation to a later paper.

In order to make an exact calculation of propagation, it is necessary to know the production cross sections of every nuclide from every heavier nuclide in interactions in the medium. In the past the required values have been predicted by using the semiempirical fits to crosssection data developed by Silberberg and Tsao. ${ }^{1}$ These fits are based on data from proton bombardment of heavy targets (p- $A$ ), and depend on measurements of the yields of individual unstable isotopes, rather than on element yields. Since our measured cross sections differ appreciably from those predicted, calculations of propagation would be improved if we could replace these relatively inaccurate predicted cross sections with values based more directly on experimental data. Therefore, it is desirable to identify some relatively accurate systemat- ic description of the variations in the cross sections with projectile type so that appropriate values of the cross sections can be modeled for all projectiles. In this work we have identified possible suitable descriptions for the elemental cross sections, but have found that we have an inadequate number of independent experimental variables to allow us, as yet, to apply these descriptions with confidence to the problem of cosmic ray propagation. In particular, we cannot make a clear distinction between the dependence of the partial cross sections on charge and on energy. More recent measurements may help us to resolve this ambiguity in the near future.

We have conducted several runs with the heavy ion beams at the Lawrence Berkeley Laboratory Bevalac. These exposures were made with additional scientific objectives beyond that of determining values of the cross sections. In particular, they were designed to calibrate the response of the detectors flown by Binns et al. ${ }^{2}$ on the Third High Energy Astronomy Observatory (HEAO-3) Heavy Nuclei Experiment, to the passage of very highly charged nuclei. These detectors were exposed in near earth orbit in order to observe the rare ultraheavy $(Z>30)$ nuclei present in the cosmic radiation. The results of the calibrations of these detectors are reported elsewhere by Newport et al. ${ }^{3}$ As a consequence of this scientific objective, the detectors used during these exposures were chosen to be basically similar to those flown on HEAO-3, although modified to optimize their suitability for use with the Bevalac beams of nuclei.

\section{EXPERIMENTAL}

The configuration of detectors adopted for the Bevalac exposure is shown in Fig. 1. The beam of nuclei was incident on an external multi-wire proportional counter (MWPC) and then passed through an array of parallel plate ionization chambers, I- 1 to I-6, and a Cherenkov detector, arranged as shown in Fig. 1. The pulse heights of the signals from each detector were recorded on tape. Thin copper decoupling foils were placed between the 


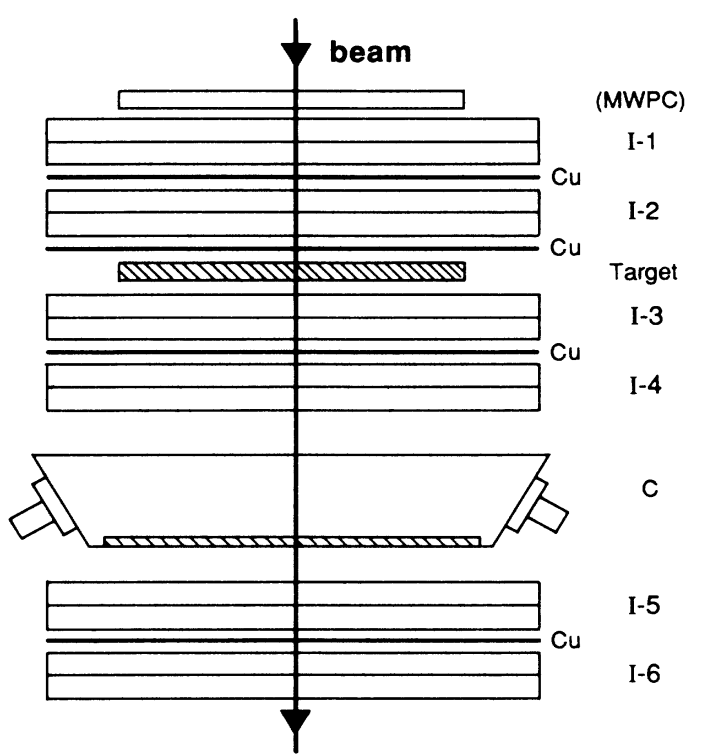

FIG. 1. Schematic configuration of the detectors used at the Bevalac.

ion chambers, while targets were inserted between I-2 and I-3 in order to study the fragments produced in them by interactions of the beam nuclei.

The beams of nuclei accelerated in the Bevalac and analyzed here were of ${ }_{26}^{56} \mathrm{Fe},{ }_{36}^{84} \mathrm{Kr},{ }_{54}^{132} \mathrm{Xe},{ }_{67}^{165} \mathrm{Ho}$, and ${ }_{79}^{197} \mathrm{Au}$, each injected at the highest readily available charge state and accelerated to the maximum rigidity of the Bevalac, giving final beam energies, after stripping, of $1.685,1.489,1.239,1.128$, and $1.049 \mathrm{GeV} /$ nucleon, respectively. These energies were measured by the bend- ing magnet current and are estimated to be accurate to $\pm 0.005 \mathrm{GeV} /$ nucleon. Exposures were made when targets of aluminum, carbon and polyethylene $\left(\mathrm{CH}_{2}\right)$ were inserted, and when there was no target present, a "blank" run. Not all the combinations of beams and targets were obtained, but Table I lists those that were, together with the numbers of incident nuclei observed in each run. The thicknesses of each target used in each run are also listed. These thicknesses were adjusted for the various beams so that in each case they were between $20 \%$ and $24 \%$ of the interaction mean free path, a value chosen as a compromise between the desirability of a high interaction rate and the necessity of applying a correction for the effects of secondary interactions in a thick target, the "thick target corrections" of Sec. III B.

\section{DATA ANALYSIS}

We used I-1 and I-2 to select those particles entering the targets which had the charge of the primary nuclei. This selection rejected the 7-10\% contamination present in each beam of nuclei with charges less than that of the beam nuclei, and also rejected a few additional secondary nuclei produced in our apparatus upstream of the targets. After making this selection we estimate, based on the resolution of I-1 and I-2, that at most there was a residual $0.5 \%$ contamination of the nuclei incident on the targets, which is partially corrected for by using the results from the appropriate blank run.

The effects on these selected incident nuclei of introducing a target were examined by making crossplots of the signals from the Cherenkov detector with the summed signals from the two ion chambers behind the target, I-3 and I-4. Such a crossplot for a run when the

TABLE I. Combinations of beams and targets used and the numbers of selected nuclei incident on the targets as observed during each run, together with the total number of interactions. The thickness of each target in each run, expressed both in $\mathrm{g} / \mathrm{cm}^{2}$ and in fractions of the mean free path (MFP) of the beam nuclei are also given. The mean energy, and spread in energy, of each beam in each target is given at the target location. Charge resolution of fragments with small $\Delta Z, 2-4$, is in charge units (c.u.) for each run.

\begin{tabular}{|c|c|c|c|c|c|c|c|}
\hline Beam & Target & $\begin{array}{c}\text { Energy in target } \\
(\mathrm{MeV} / \text { nucleon })\end{array}$ & $\left(\mathrm{g} / \mathrm{cm}^{2}\right)$ & $\begin{array}{l}\text { Thickness } \\
\text { fraction of } \text { MFP }^{\mathrm{a}}\end{array}$ & $\begin{array}{c}\text { Incident } \\
\text { on target }\left(\times 10^{3}\right)\end{array}$ & $\begin{array}{c}\text { No. of } \\
\text { interactions }\end{array}$ & $\begin{array}{c}\text { Charge } \\
\text { resolution (c.u.) }\end{array}$ \\
\hline iron & blank & 1615 & & & 148 & & \\
\hline $\begin{array}{l}\text { krypton } \\
\text { krypton }\end{array}$ & $\begin{array}{l}\text { aluminum } \\
\text { blank }\end{array}$ & $\begin{array}{l}1419 \pm 55 \\
1474\end{array}$ & 4.25 & 0.22 & $\begin{array}{l}86 \\
33\end{array}$ & 11501 & 0.19 \\
\hline $\begin{array}{l}\text { xenon } \\
\text { xenon } \\
\text { xenon } \\
\text { xenon }\end{array}$ & $\begin{array}{l}\text { aluminum } \\
\text { carbon } \\
\text { polyethylene } \\
\text { blank }\end{array}$ & $\begin{array}{l}1155 \pm 62 \\
1175 \pm 41 \\
1190 \pm 27 \\
1237\end{array}$ & $\begin{array}{l}3.29 \\
1.94 \\
1.08\end{array}$ & $\begin{array}{l}0.20 \\
0.22 \\
0.21\end{array}$ & $\begin{array}{r}62 \\
98 \\
148 \\
62\end{array}$ & $\begin{array}{l}11831 \\
19253 \\
28148\end{array}$ & $\begin{array}{l}0.23 \\
0.19 \\
0.19\end{array}$ \\
\hline $\begin{array}{l}\text { holmium } \\
\text { holmium } \\
\text { holumium } \\
\text { holmium }\end{array}$ & $\begin{array}{l}\text { aluminum } \\
\text { carbon } \\
\text { polyethylene } \\
\text { blank }\end{array}$ & $\begin{array}{l}1022 \pm 78 \\
1048 \pm 52 \\
1067 \pm 34 \\
1100\end{array}$ & $\begin{array}{l}3.29 \\
1.94 \\
1.08\end{array}$ & $\begin{array}{l}0.22 \\
0.24 \\
0.24\end{array}$ & $\begin{array}{r}160 \\
111 \\
171 \\
65\end{array}$ & $\begin{array}{l}32634 \\
24340 \\
35722\end{array}$ & $\begin{array}{l}0.23 \\
0.26 \\
0.25\end{array}$ \\
\hline $\begin{array}{l}\text { gold } \\
\text { gold } \\
\text { gold } \\
\text { gold }\end{array}$ & $\begin{array}{l}\text { aluminum } \\
\text { carbon } \\
\text { polyethylene } \\
\text { blank }\end{array}$ & $\begin{array}{r}932 \pm 83 \\
961 \pm 55 \\
982 \pm 34 \\
1016\end{array}$ & $\begin{array}{l}2.95 \\
1.73 \\
0.92\end{array}$ & $\begin{array}{l}0.22 \\
0.23 \\
0.22\end{array}$ & $\begin{array}{l}160 \\
205 \\
296 \\
194\end{array}$ & $\begin{array}{l}30767 \\
43291 \\
58518\end{array}$ & $\begin{array}{l}0.28 \\
0.30 \\
0.26\end{array}$ \\
\hline
\end{tabular}

${ }^{\text {a }}$ Based on Eq. (11). 
target was present was compared with a similar crossplot for a blank run. Fig. 2(a) and 2(b) show such a comparison for the case of xenon nuclei incident on a carbon target. The individual fragments produced in the target are clearly identified and well resolved, lying along the diagonal band in Fig. 2(a). Also to be seen in this figure are the interactions in the Cherenkov detector, lying along the horizontal band, with the region between these two bands populated by beam particle interactions in the ion chambers and copper decouplers, together with fragments from secondary interactions in the detectors. The relative scarcity of events in the diagonal fragment band in the plot for the blank run shows that the corrections for secondary interactions were quite minor. The charge resolution achieved by using the Cherenkov detector response alone and selecting along the fragment

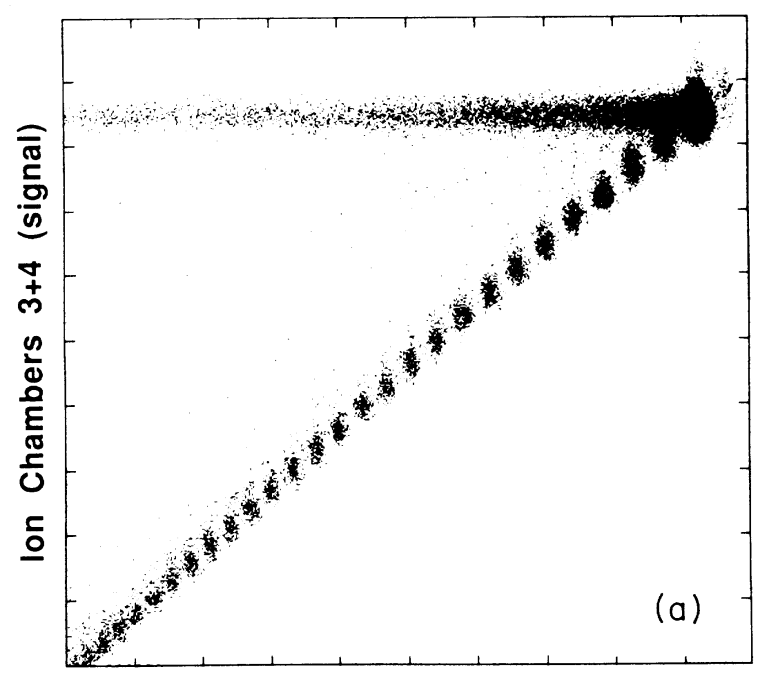

Cherenkov Signal

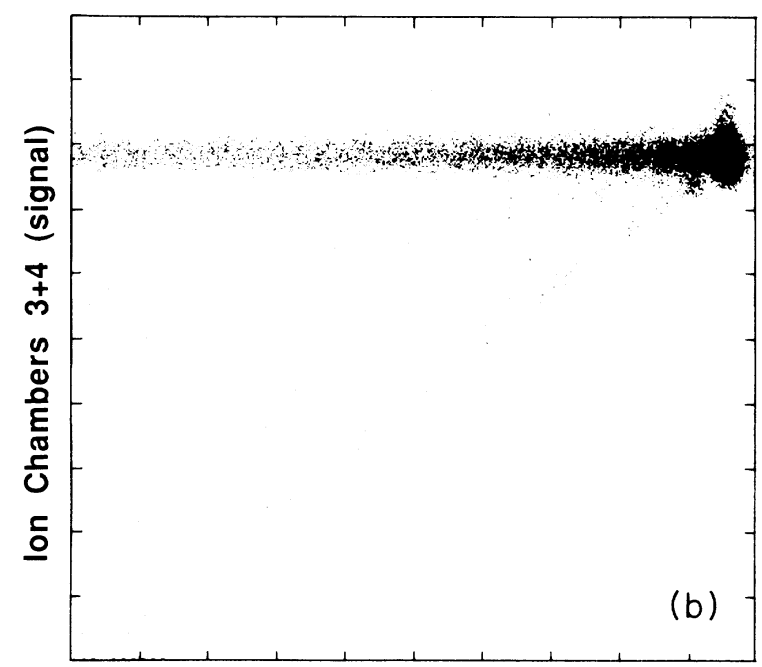

Cherenkov Signal

FIG. 2. (a) Plot, over the relevant ranges, of signals from the Cherenkov detector, against the sum of those from the ion chambers mounted behind the target, I-3 and I-4, for the example of xenon nuclei incident on a carbon target. (b) As in (a) but for the xenon blank run.

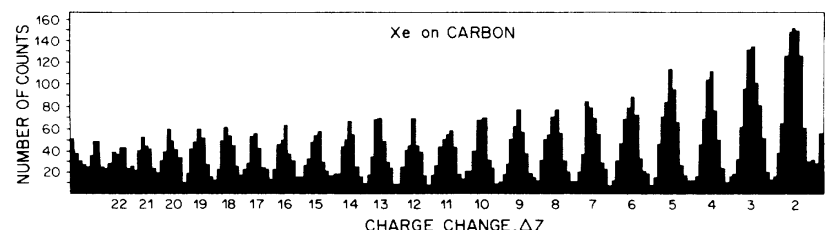

FIG. 3. The charge spectrum derived for fragments of xenon nuclei incident on a carbon target derived from the data shown in Fig. 2(a).

band in Fig. 2(a) is illustrated in Fig. 3. Table I lists the charge resolution, in charge units (c.u.) achieved by using both the Cherenkov detector and the ion chambers for each beam, measured from the width of the peaks of the fragments with small charge changes $(2 \leq \Delta Z \leq 4)$.

\section{A. Total cross sections}

The total charge changing cross-section, $\sigma_{\text {tot }}\left(Z_{B}, Z_{T}\right)$, which is a function of both the beam charge $Z_{B}$ and the target charge $Z_{T}$, is determined from the fraction of beam nuclei that survive passage through the target and the Cherenkov detector. In order to correct for interactions in the detectors we used the blank runs. Histograms of the signals from the Cherenkov detector for these blank runs showed a large peak due to surviving beam nuclei and a tail of smaller signals due to interactions in the detectors, mainly in the Cherenkov detector itself. These histograms, an example of which is shown in Fig. 4, were fit with a Gaussian plus a small background function that exponentially rises to the peak and is then terminated with a Gaussian tail. The number of nuclei surviving through the Cherenkov detector, $N_{\text {obs }}^{\text {blank }}$, was found from the deduced number in the peak. For the target runs, similar histograms were fit to two Gaussians, one for the beam and one for the $\Delta Z=1 \mathrm{nu}$ clei, since these were generally not well resolved, together with a background scaled from the blank run. The numbers of surviving nuclei, $N_{\text {obs }}^{T}$ or $N_{\text {obs }}^{\text {blank }}$, numbers of unresolved fragments with a charge change, $\Delta Z$, of one, $\delta N_{\Delta Z=1}$, and the corrections for background, $N_{\text {back }}$, are given in Table II for all beams and targets.

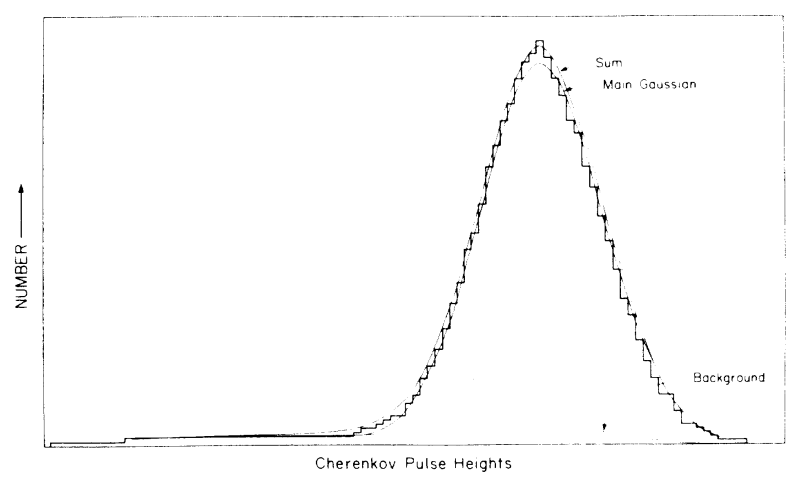

FIG. 4. A histogram of the signals from the Cherenkov detector for xenon nuclei in the blank run, plotted with varying bin sizes to smooth the data, showing the Gaussian and background functions fitted to the data. 
TABLE II. Values of the various corrections made to the raw data in determining the partial cross-sections. The numbers of nuclei incident on the targets or in the blank runs are shown by $N_{B}^{T}$ or $N_{B}^{\text {blank }}$, respectively. The corrections for charge changes of 1 and for background are given as $\delta N_{\Delta Z=1}$ and $N_{\text {back }}$ respectively, together with the resulting percentage corrections.

\begin{tabular}{|c|c|c|c|c|c|c|c|c|c|}
\hline \multirow[b]{2}{*}{ Beams } & \multirow[b]{2}{*}{ Targets } & \multirow{2}{*}{$\begin{array}{l}\text { Incident } \\
N_{B}^{T} \\
\end{array}$} & \multirow{2}{*}{$\begin{array}{c}\text { on target } \\
N_{B}^{\text {blank }}\end{array}$} & \multirow{2}{*}{$\begin{array}{l}\text { No. out of } \\
\text { Cherenkov }\end{array}$} & \multirow[b]{2}{*}{$\delta N_{\Delta Z=1}$} & \multirow[b]{2}{*}{$N_{\text {back }}$} & \multirow[b]{2}{*}{$\%$ corr. } & \multicolumn{2}{|c|}{$\begin{array}{l}\text { Corrected no. } \\
\text { out of Cherenkov }\end{array}$} \\
\hline & & & & & & & & $N_{\text {obs }}^{T}$ & $N_{\mathrm{obs}}^{\text {blank }}$ \\
\hline krypton & aluminum & 86104 & & 60198 & 48 & 2068 & 3.52 & 58082 & \\
\hline krypton & blank & & 33369 & 29169 & & 935 & 3.21 & & 28234 \\
\hline xenon & aluminum & 61936 & & 42378 & 2 & 2365 & 5.59 & 40011 & \\
\hline xenon & carbon & 98299 & & 66539 & 43 & 4309 & 6.54 & 62187 & \\
\hline xenon & polyethylene & 148269 & & 101227 & 118 & 6691 & 6.73 & 94418 & \\
\hline xenon & blank & & 62439 & 52495 & & 3413 & 6.50 & & 49082 \\
\hline holmium & aluminum & 159577 & & 105318 & 126 & 9142 & 8.80 & 96050 & \\
\hline holmium & carbon & 111035 & & 72074 & 266 & 6213 & 8.99 & 65595 & \\
\hline holmium & polyethylene & 171333 & & 112557 & 488 & 9600 & 8.96 & 102469 & \\
\hline holmium & blank & & 64583 & 53400 & & 4531 & 8.49 & & 48869 \\
\hline gold & aluminum & 160277 & & 107113 & 720 & 11767 & 11.66 & 94626 & \\
\hline gold & carbon & 205171 & & 134322 & 1128 & 14824 & 11.88 & 118370 & \\
\hline gold & polyethylene & 295571 & & 196356 & 1887 & 21310 & 11.81 & 173159 & \\
\hline gold & blank & & 193980 & 159475 & & 17739 & 11.12 & & 141739 \\
\hline
\end{tabular}

The interaction mean free paths of the beam nuclei in the targets are determined from the relation

$N_{\text {obs }}^{T}=N_{B}^{T} \exp \left(-x_{t} / \lambda_{t}\right) \exp \left(-x_{\text {IC }} / \lambda_{\text {IC }}\right) \exp \left(-x_{C} / \lambda_{C}\right)$,

where $N_{B}^{T}$ is the number of beam nuclei entering the target as determined by the initial selection, the $x$ 's are the thicknesses of the various materials, the $\lambda$ 's are the mean free paths of the beam in those materials and the subscripts $t, \mathrm{IC}$, and $C$ refer to the target, ion chambers 3 and 4 , and the Cherenkov detector, respectively. The corresponding expression for the blank run is

$$
N_{\text {obs }}^{\text {blank }}=N_{B}^{\text {blank }} \exp \left(-x_{\text {IC }} / \lambda_{\text {IC }}\right) \exp \left(-x_{C} / \lambda_{C}\right),
$$

where the notation is the same as for Eq. (1). Taking the ratio of Eq. (1) to Eq. (2) gives

$$
N_{\text {obs }}^{T} / N_{\text {obs }}^{\text {blank }}=\left[N_{B}^{T} \exp \left(-x_{t} / \lambda_{t}\right)\right] /\left(N_{B}^{\text {blank }}\right)
$$

which cancels the terms due to the ion chambers and the Cherenkov detector. Rearranging gives

$$
\lambda_{t}=x_{t} / \ln \left[\left(N_{B}^{T} / N_{\mathrm{obs}}^{T}\right)\left(N_{\mathrm{obs}}^{\mathrm{blank}} / N_{B}^{\text {blank }}\right)\right] .
$$

The total interaction cross section per nucleus, $\sigma_{\text {tot }}$, is related to the mean free path by

$$
\sigma_{\text {tot }}=\left\langle A^{T}\right\rangle /\left(6.02 \times 10^{-4}\right) \lambda_{t},
$$

where $\left\langle A^{T}\right\rangle$ is the average mass number of the target nuclei and the units are chosen so that if $\lambda$ is expressed in $\mathrm{g} / \mathrm{cm}^{2}$, then $\sigma_{\text {tot }}$ is in $\mathrm{mb}$. The cross sections measured in carbon and polyethylene allow us to deduce those in hydrogen from:

$$
\sigma_{\mathrm{H}}=0.5\left\{3\left\langle\sigma_{\text {poly }}\right\rangle-\sigma_{\mathrm{C}}\right\}
$$

\begin{tabular}{|c|c|c|c|c|c|c|c|c|c|}
\hline \multirow[b]{2}{*}{ Beams } & \multirow[b]{2}{*}{ Targets } & \multicolumn{2}{|c|}{$Z+1$ correction } & \multicolumn{2}{|c|}{ Blank correction } & \multicolumn{2}{|c|}{ Absorp. correction } & \multicolumn{2}{|c|}{ Thick target correction } \\
\hline & & Small $\Delta Z$ & Large $\Delta Z$ & Small $\Delta Z$ & Large $\Delta Z$ & Small $\Delta Z$ & Large $\Delta Z$ & Small $\Delta Z$ & Large $\Delta Z$ \\
\hline krypton & aluminum $^{\mathrm{a}}$ & 1.3 & 1.0 & 4.0 & 2.7 & 15.4 & 11.4 & 8.9 & 1.9 \\
\hline xenon & aluminum & 0.3 & 1.7 & 6.3 & 1.0 & 19.5 & 13.6 & 8.5 & 1.8 \\
\hline xenon & carbon & 1.0 & 1.7 & 5.3 & 2.3 & 19.9 & 14.4 & 9.1 & 0.7 \\
\hline xenon & polyethylene & 1.0 & 1.0 & 3.3 & 3.0 & 19.6 & 13.5 & 6.8 & 0.0 \\
\hline holmium & aluminum & 1.0 & 1.0 & 5.3 & 1.0 & 22.5 & 17.0 & 9.3 & 1.0 \\
\hline holmium & carbon & 1.0 & 2.0 & 6.0 & 1.3 & 22.7 & 16.8 & 9.2 & 0.4 \\
\hline holmium & polyethylene & 1.7 & 1.7 & 3.7 & 3.3 & 22.4 & 16.8 & 7.7 & 0.0 \\
\hline gold & aluminum & 3.7 & 1.7 & 7.3 & 1.0 & 25.1 & 19.6 & 8.2 & 1.6 \\
\hline gold & carbon & 3.7 & 3.0 & 6.3 & 2.3 & 25.1 & 19.8 & 9.1 & 1.2 \\
\hline gold & polyethylene & 4.0 & 3.0 & 3.7 & 4.3 & 25.1 & 19.7 & 7.8 & 0.0 \\
\hline
\end{tabular}

TABLE III. Characteristic values of the various corrections made to the data in determining the partial cross-sections for small $\Delta Z(=2-4)$ and large $\Delta Z(=25-27)$. Each correction, described in Sec. III B, is expressed as a percentage of the value being corrected.

${ }^{\text {a }}$ Large $\Delta Z=16-18$. 
where $\left\langle\sigma_{\text {poly }}\right\rangle$ is the average cross section per nucleus in the $\mathrm{CH}_{2}$ molecule.

\section{B. Partial cross sections}

Partial cross sections are determined from the numbers of nuclei that are fragments which are produced in the target and survive through the detectors. These observed numbers of fragment nuclei are derived from histograms such as Fig. 3 by simply counting the numbers of events between valleys in the distributions and thus include any contribution from backgrounds due to multiple fragments, etc. The observed numbers have to be corrected for various effects in order to derive the cross sections. For a fragment of a given charge there is a contribution due to fragments of the next higher charge interacting in the Cherenkov detector, since the ion chamber resolution was insufficient to resolve charge changes of $\Delta Z=1$. Also, as seen from the crossplots for the blank runs, Fig. 2(b), some nuclei result from interactions of the beam in I-2, the copper decoupler and I-3. In both cases the corrections for these effects, referred to as the $(Z+1)$ and blank backgrounds are quite small and the details are described elsewhere by Kertzman. ${ }^{4}$ Representative values of these corrections are given in Table III for each run.

The numbers of fragment nuclei corrected for these backgrounds have then to be corrected for the effects of absorption in the detector and for possible multiple interactions in the target. Absorption of the fragments in the detector was corrected for using an exponential absorption law:

$$
N_{\text {exit }}=N_{\text {obs }} \exp \left[\Sigma\left(x_{i} / \lambda_{i}\right)\right],
$$

where $N_{\text {exit }}$ is the number of fragments emerging from the target, $N_{\text {obs }}$ is the number of fragments observed in the Cherenkov detector, the $x_{i}$ 's are the thicknesses of the various detector materials, and the $\lambda_{i}$ 's are the mean free paths of each fragment in these materials, which are found from the total cross section formula of Eq. (11) derived from our own measurements (see Sec. IV A).

If the targets had been very thin then the cross sec- tions for the production of fragments with a charge change of $\Delta Z$ from the primary charge, $Z, \sigma(\Delta Z)$, could have been found from the expression

$$
\sigma(\Delta Z) / \sigma_{\text {tot }}=N_{\text {exit }}(\Delta Z) / N_{I},
$$

where $N_{\text {exit }}(\Delta Z)$ is the number of fragments of charge $\left(Z_{B}-\Delta Z\right)$ leaving the target and $N_{I}$ the number of beam nuclei that interact in the target. However, the targets used were all between $20 \%$ and $24 \%$ of the beam mean free path (Table I) and hence some of the fragments produced will interact before they leave the target. In order to correct for these "thick" targets a standard slab propagation program was used. ${ }^{4}$ This program requires estimates for the values of all the partial cross sections over the range of charges being considered, and, from the number of nuclei assumed to be incident, calculates the number leaving. Initial estimates of $\sigma(\Delta Z)$ from our measurements were based on Eq. (8) and the intermediate $\sigma(\Delta Z)$ values calculated assuming that the ratio of the partial cross sections to the total cross sections for a given $\Delta Z$ is constant for all $Z$ in this range of $Z$. These estimates were then iterated by the calculated values for the numbers of fragments exiting the target until these calculated numbers agreed with those observed to within $1 \%$. The magnitude of the resulting thick target corrections are shown in Table III, where it can be seen that they are as great as $9 \%$ for small $\Delta Z$ but become negligible for large charge changes.

A check on the validity of this procedure was obtained from the straightforward calculation of the corrections needed for a charge change of 1 or 2 , which can be readily expressed in simple analytical form. This check showed that the iterative procedure gave results valid to within the expected $1 \%$.

\section{RESULTS}

\section{A. Total charge changing cross sections}

The values determined for the total charge changing cross-section, $\sigma_{\text {tot }}$, for each beam and target combination

TABLE IV. Total charge changing cross sections for each beam and each target. Also given are the values of the fitting param-

\begin{tabular}{|c|c|c|c|c|c|c|c|}
\hline Beams & Targets & $\begin{array}{l}\text { Charge changing } \\
\text { cross sections }(\mathrm{mb})\end{array}$ & $\sigma_{0}(\mathrm{mb})$ & $\begin{array}{l}\text { Fitting } \\
\Sigma_{0}(\mathrm{mb})\end{array}$ & $\begin{array}{c}\text { Parameters } \\
\Delta_{0} \\
\end{array}$ & $\alpha$ & $\begin{array}{c}\text { Reduced } \\
\chi^{2} \\
\end{array}$ \\
\hline krypton & aluminum & $2329 \pm 102$ & & $163.0 \pm 7.2$ & & $0.530 \pm 0.021$ & 0.82 \\
\hline xenon & aluminum & $2890 \pm 120$ & & $187.5 \pm 10.2$ & & $0.587 \pm 0.026$ & 0.98 \\
\hline xenon & carbon & $2240 \pm 82$ & & $198.9 \pm 7.7$ & & $0.654 \pm 0.018$ & 1.15 \\
\hline xenon & polyethylene & $1511 \pm 86$ & $159.7 \pm 2.9$ & & $7.63 \pm 0.11$ & & 2.15 \\
\hline xenon & hydrogen & $1146 \pm 89$ & $192.3 \pm 5.7$ & & $6.29 \pm 0.15$ & & 2.26 \\
\hline holmium & aluminum & $3120 \pm 100$ & & $226.8 \pm 10.4$ & & $0.660 \pm 0.021$ & 0.87 \\
\hline holmium & carbon & $2564 \pm 70$ & & $229.6 \pm 8.6$ & & $0.702 \pm 0.017$ & 0.79 \\
\hline holmium & polyethylene & $1693 \pm 72$ & $174.8 \pm 3.0$ & & $7.46 \pm 0.11$ & & 1.46 \\
\hline holmium & hydrogen & $1258 \pm 75$ & $206.0 \pm 5.8$ & & $6.29 \pm 0.15$ & & 2.71 \\
\hline gold & aluminum & $3240 \pm 82$ & & $244.6 \pm 12.1$ & & $0.721 \pm 0.022$ & 0.82 \\
\hline gold & carbon & $2731 \pm 58$ & & $221.0 \pm 9.2$ & & $0.703 \pm 0.019$ & 1.16 \\
\hline gold & polyethylene & $1861 \pm 63$ & $202.7 \pm 3.7$ & & $6.54 \pm 0.08$ & & 2.91 \\
\hline gold & hydrogen & $1426 \pm 65$ & $282.9 \pm 8.3$ & & $5.10 \pm 0.10$ & & 2.40 \\
\hline
\end{tabular}
eters for the partial cross sections, see Sec. IV B, Eqs. (12) and (13), together with the values of reduced $\chi^{2}$ in each case. 
are given in Table IV. Extensive earlier measurements with lighter beam nuclei $(Z \leq 26)$ by Westfall et al. ${ }^{5}$ and others, have suggested that there is no significant energy dependence in $\sigma_{\text {tot }}$ over energies ranging from a few hundred $\mathrm{MeV} /$ nucleon to about two $\mathrm{GeV} /$ nucleon, unlike the case for the partial cross sections. We have consequently followed previous work in assuming that $\sigma_{\text {tot }}$ is independent of energy and depends only on the mass numbers of the target and projectile nuclei. The extensive data reported for $Z_{B} \leq 26$ by Westfall et al. ${ }^{5}$ was used by them to derive an expression:

$$
\sigma_{\mathrm{W}}\left(A_{B}, A_{T}\right)=10 \pi(1.35)^{2}\left(A_{T}^{1 / 3}+A_{B}^{1 / 3}-0.83\right)^{2} \mathrm{mb}
$$

for nucleus-nucleus collisions, arbitrarily setting $A_{T}=0.089$ for the case of a hydrogen target. This relation basically assumes that the overlap required before a charge changing interaction occurs is mass independent and that a nucleus has a radius given by $r_{0} A^{1 / 3}$, where $r_{0}=1.35 \times 10^{-15} \mathrm{~m}$. An earlier expression proposed by Hagen $^{6}$ fits most of the same data by the relation

$$
\sigma_{\mathrm{Ha}}=10 \pi(1.29)^{2}\left\{A_{T}^{1 / 3}+A_{B}^{1 / 3}-1.189 \exp \left[-0.05446 \min \left(A_{T}, A_{B}\right)\right]\right\}^{2} \mathrm{mb}
$$

which assumes a mass dependent overlap, where $\min \left(A_{T}, A_{B}\right)$ is the lesser of $A_{T}$ or $A_{B}$. In this case $A_{T}$ for hydrogen was simply set equal to 1 , and there is not a good fit to the hydrogen data.

Neither of these relations extrapolates well to fit the data in the mass region covered in this work. Instead we fit our data to a relation of the form

$$
\sigma_{\mathrm{K}}=10 \pi(1.35)^{2}\left[A_{T}^{1 / 3}+A_{B}^{1 / 3}-p\left(A_{T}+A_{B}\right)^{q}\right]^{2} \mathrm{mb},
$$

where $p$ and $q$ were initially arbitrary constants formally determined from the data to have values of $p=0.209$ and $q=0.332$. In practice, these constants are not independent and quite a wide range of values give equally good values of $\chi^{2}$. If $q$ is set equal to $1 / 3$ then $p=0.209 \pm 0.002$. Reasonable values of $\chi^{2}$ could not be obtained to a fit to an equation like Eq. (9) with a constant overlap term. Like Westfall et al., ${ }^{5}$ we had to assume that for hydrogen we should set $A_{T}=0.089$ in making the fit to the data. This relation also gives a good fit to the data of Westfall et al. ${ }^{5}$ on Fe nuclei for our light targets where $A_{T} \ll A_{B}$, but does not match the data for still lower beam charges, for which Eq. (9) is better. Physically, this expression corresponds to an overlap model with the overlap dependent on the masses of both nuclei involved, unlike the case for either of the earlier expressions. ${ }^{5,6}$

Our measured cross sections are compared with those predicted by these various expressions in Figs. 5(a) and 5(b). It can be seen that both Eqs. (9) and (10) tend to overestimate the cross sections of the heavy nuclei by
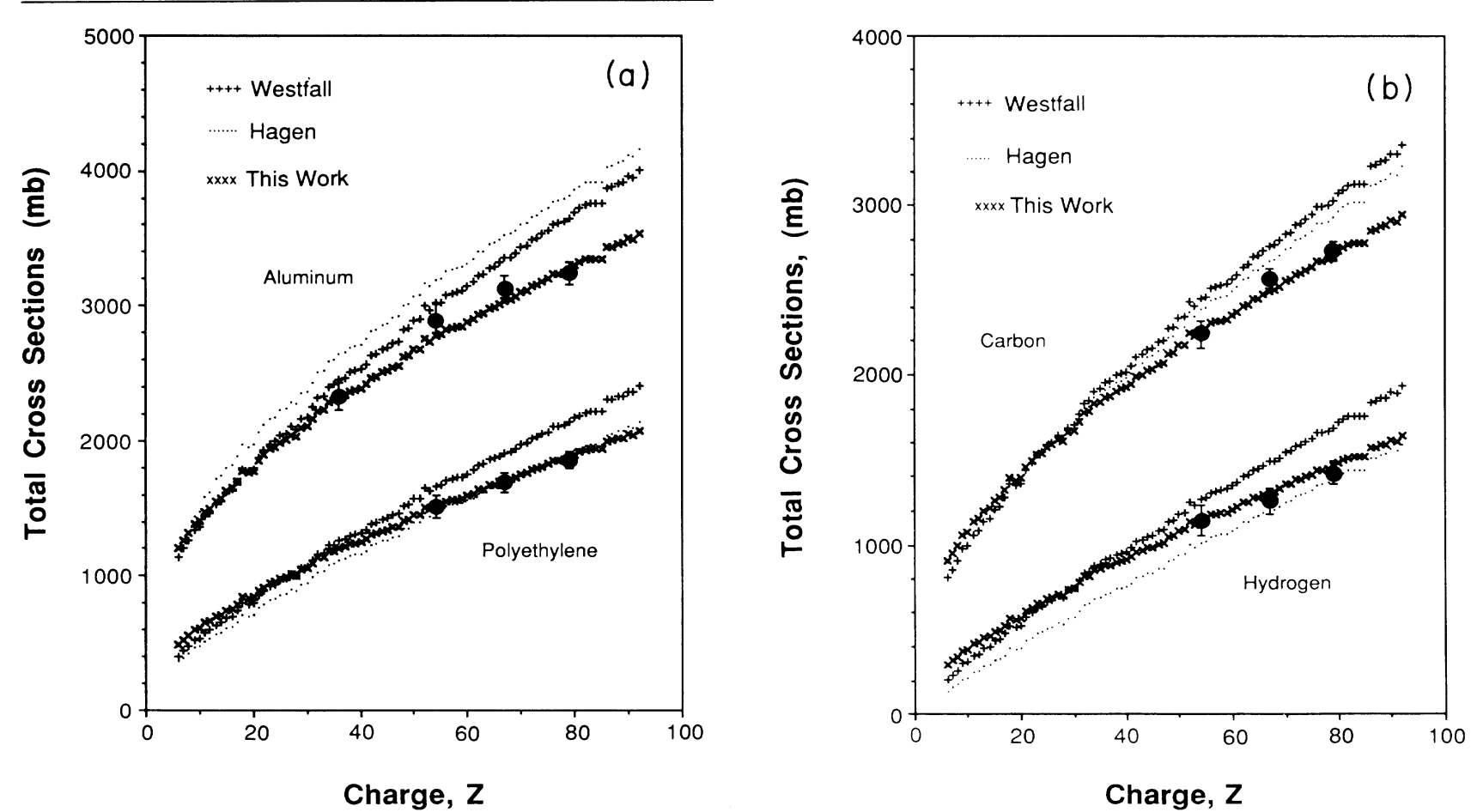

FIG. 5. (a) Calculated total cross sections in aluminum and polyethylene targets for the most abundant or longest lived isotope of each element between carbon and uranium. Also shown are our measured values with error bars. (b) As in (a) but for carbon and hydrogen targets. 
some $10-20 \%$, but that our expression converges with them around iron. It should be noted that our relation was derived only from our measured values and the fact that it extrapolates to the iron values so well is encouraging. All these relations show a closely similar dependence on $Z$, so that comparative cross-section estimates made over a limited range of charges, calculated with any one will not be seriously in error.

\section{B. Partial cross-sections systematics}

The partial cross sections determined for each beam and each target studied are listed in Tables V-VIII. In every case the majority of the values of the partial cross sections are found to regularly decrease with increasing charge change. As an example, Fig. 6 shows the variation of $\sigma(\Delta Z)$ with $\Delta Z$, plotted on linear scales, for holmium nuclei on carbon and hydrogen targets. It can be seen that while in each case there is a smooth and regular decrease, the form of this decrease is quite different in the two examples. In fact, in all cases we find that the variation of $\sigma(\Delta Z)$ can, depending on the type of target material, be quite well represented by either a power law or exponential law relation between $\sigma(\Delta Z)$ and $\Delta Z$. Examples are given in Figs. 7-10, which show several

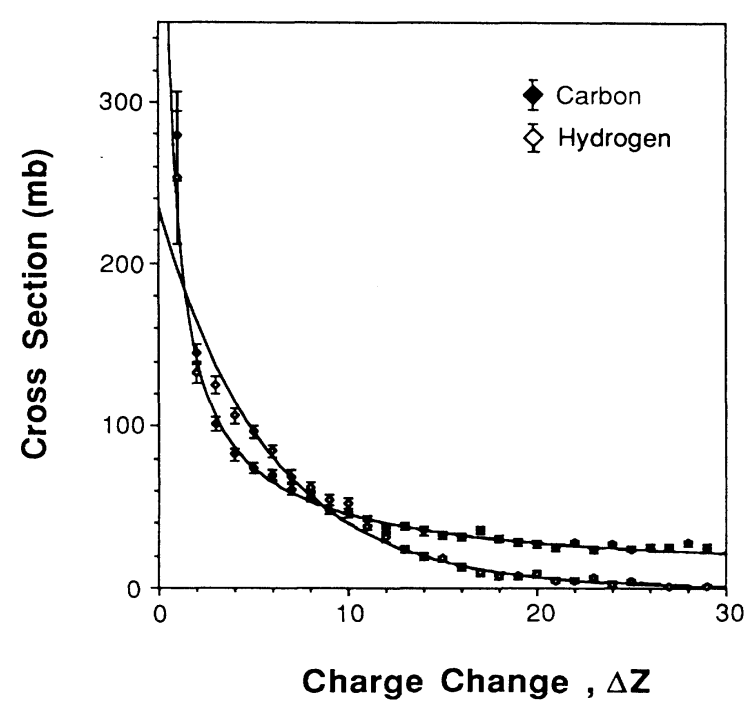

FIG. 6. The variation of the partial cross sections, $\sigma(\Delta Z)$, for holmium nuclei on carbon and hydrogen targets, plotted on linear scales. Also shown is the best fit for $2 \leq \Delta Z \leq 20$.

TABLE V. Cross sections of gold in various media.

\begin{tabular}{lccccc}
\hline \hline & \multicolumn{5}{c}{ TABLen } \\
$Z$ & $\Delta Z$ & $\begin{array}{c}\text { Aluminum } \\
(\mathrm{mb})\end{array}$ & $\begin{array}{c}\text { Carbon } \\
(\mathrm{mb})\end{array}$ & $\begin{array}{c}\text { Polyethylene } \\
(\mathrm{mb})\end{array}$ & $\begin{array}{c}\text { Hydrogen } \\
(\mathrm{mb})\end{array}$ \\
\hline 78 & 1 & $313.64 \pm 31.02$ & $270.90 \pm 26.90$ & $253.90 \pm 25.50$ & $245.40 \pm 40.50$ \\
77 & 2 & $161.90 \pm 15.93$ & $144.20 \pm 14.40$ & $170.30 \pm 17.10$ & $183.40 \pm 26.60$ \\
76 & 3 & $112.31 \pm 4.97$ & $105.50 \pm 3.80$ & $134.70 \pm 3.30$ & $149.40 \pm 5.30$ \\
75 & 4 & $86.05 \pm 4.17$ & $78.90 \pm 3.10$ & $107.80 \pm 2.80$ & $122.30 \pm 4.50$ \\
74 & 5 & $83.02 \pm 3.94$ & $75.30 \pm 3.00$ & $93.00 \pm 2.50$ & $101.80 \pm 4.00$ \\
73 & 6 & $60.11 \pm 3.53$ & $56.30 \pm 2.60$ & $80.20 \pm 2.30$ & $92.10 \pm 3.60$ \\
72 & 7 & $58.86 \pm 3.20$ & $54.30 \pm 2.40$ & $73.30 \pm 2.10$ & $82.90 \pm 3.40$ \\
71 & 8 & $52.80 \pm 3.00$ & $53.80 \pm 2.40$ & $59.10 \pm 1.80$ & $61.70 \pm 3.00$ \\
70 & 9 & $50.64 \pm 2.86$ & $47.80 \pm 2.20$ & $54.40 \pm 1.70$ & $57.70 \pm 2.80$ \\
69 & 10 & $46.91 \pm 2.71$ & $47.60 \pm 2.20$ & $42.60 \pm 1.50$ & $40.10 \pm 2.50$ \\
68 & 11 & $47.38 \pm 2.79$ & $41.70 \pm 2.10$ & $34.40 \pm 1.30$ & $30.70 \pm 2.30$ \\
67 & 12 & $41.46 \pm 2.58$ & $40.10 \pm 2.00$ & $30.90 \pm 1.30$ & $26.40 \pm 2.20$ \\
66 & 13 & $41.19 \pm 2.53$ & $36.20 \pm 1.90$ & $26.00 \pm 1.10$ & $20.90 \pm 1.90$ \\
65 & 14 & $37.47 \pm 2.38$ & $31.90 \pm 1.80$ & $22.60 \pm 1.10$ & $17.90 \pm 1.80$ \\
64 & 15 & $33.93 \pm 2.28$ & $32.30 \pm 1.80$ & $19.90 \pm 1.00$ & $13.70 \pm 1.70$ \\
63 & 16 & $33.97 \pm 2.24$ & $31.10 \pm 1.70$ & $17.40 \pm 0.90$ & $10.60 \pm 1.60$ \\
62 & 17 & $30.60 \pm 2.15$ & $30.20 \pm 1.70$ & $16.70 \pm 0.90$ & $9.90 \pm 1.60$ \\
61 & 18 & $29.64 \pm 2.09$ & $29.70 \pm 1.70$ & $12.80 \pm 0.80$ & $4.40 \pm 1.50$ \\
60 & 19 & $27.88 \pm 1.99$ & $28.10 \pm 1.60$ & $12.90 \pm 0.90$ & $5.40 \pm 1.40$ \\
59 & $20^{\mathrm{a}}$ & $28.58 \pm 2.04$ & $26.80 \pm 1.60$ & $12.50 \pm 0.90$ & $5.40 \pm 1.40$ \\
58 & $21^{\mathrm{a}}$ & $32.33 \pm 2.15$ & $27.00 \pm 1.60$ & $11.00 \pm 0.70$ & $3.00 \pm 1.40$ \\
57 & $22^{\mathrm{a}}$ & $30.29 \pm 2.08$ & $30.60 \pm 1.70$ & $14.70 \pm 0.80$ & $6.70 \pm 1.50$ \\
56 & $23^{\mathrm{a}}$ & $28.40 \pm 2.00$ & $29.40 \pm 1.60$ & $15.60 \pm 0.80$ & $8.70 \pm 1.50$ \\
55 & $24^{\mathrm{a}}$ & $34.41 \pm 2.19$ & $32.30 \pm 1.70$ & $17.00 \pm 0.90$ & $9.40 \pm 1.60$ \\
54 & $25^{\mathrm{a}}$ & $37.77 \pm 2.32$ & $32.40 \pm 1.70$ & $18.50 \pm 0.90$ & $11.60 \pm 1.60$ \\
53 & $26^{\mathrm{a}}$ & $30.97 \pm 2.08$ & $26.30 \pm 1.50$ & $16.30 \pm 0.90$ & $11.20 \pm 1.50$ \\
52 & $27^{\mathrm{a}}$ & $27.96 \pm 1.98$ & $28.00 \pm 1.60$ & $15.00 \pm 0.80$ & $8.50 \pm 1.50$ \\
51 & $28^{\mathrm{a}}$ & $30.08 \pm 2.03$ & $22.40 \pm 1.40$ & $13.00 \pm 0.80$ & $8.30 \pm 1.40$ \\
50 & $29^{\mathrm{a}}$ & $26.78 \pm 1.92$ & $25.30 \pm 1.50$ & $12.50 \pm 0.80$ & $6.00 \pm 1.40$ \\
\hline \hline
\end{tabular}

${ }^{a}$ Values include an additional component due to fission, see Sec. IV D. 
TABLE VI. Cross sections of holmium in various media.

\begin{tabular}{|c|c|c|c|c|c|}
\hline$\underline{Z}$ & $\Delta Z$ & $\begin{array}{c}\text { Aluminum } \\
(\mathrm{mb})\end{array}$ & $\begin{array}{c}\text { Carbon } \\
(\mathrm{mb})\end{array}$ & $\begin{array}{c}\text { Polyethylene } \\
(\mathrm{mb})\end{array}$ & $\begin{array}{c}\text { Hydrogen } \\
(\mathrm{mb})\end{array}$ \\
\hline 66 & 1 & $258.2 \pm 26.5$ & $278.6 \pm 28.1$ & $261.4 \pm 26.3$ & $252.8 \pm 41.8$ \\
\hline 65 & 2 & $157.1 \pm 15.6$ & $144.7 \pm 5.5$ & $136.6 \pm 3.6$ & $132.5 \pm 6.0$ \\
\hline 64 & 3 & $107.6 \pm 4.5$ & $101.8 \pm 4.4$ & $117.7 \pm 3.2$ & $125.6 \pm 5.3$ \\
\hline 63 & 4 & $88.9 \pm 3.8$ & $82.5 \pm 3.8$ & $98.5 \pm 2.8$ & $106.5 \pm 4.6$ \\
\hline 62 & 5 & $82.4 \pm 3.7$ & $74.1 \pm 3.5$ & $89.0 \pm 2.6$ & $96.5 \pm 4.3$ \\
\hline 61 & 6 & $65.7 \pm 3.2$ & $69.6 \pm 3.4$ & $79.6 \pm 2.4$ & $84.6 \pm 4.0$ \\
\hline 60 & 7 & $63.5 \pm 3.2$ & $61.0 \pm 3.2$ & $66.3 \pm 2.2$ & $69.0 \pm 3.7$ \\
\hline 59 & 8 & $58.4 \pm 3.0$ & $56.5 \pm 3.0$ & $60.1 \pm 2.1$ & $61.9 \pm 3.4$ \\
\hline 58 & 9 & $52.5 \pm 2.8$ & $48.5 \pm 2.8$ & $52.6 \pm 1.9$ & $54.7 \pm 3.2$ \\
\hline 57 & 10 & $51.6 \pm 2.8$ & $46.9 \pm 2.7$ & $50.6 \pm 1.9$ & $52.5 \pm 3.1$ \\
\hline 56 & 11 & $44.4 \pm 2.6$ & $42.4 \pm 2.7$ & $39.9 \pm 1.7$ & $38.6 \pm 2.9$ \\
\hline 55 & 12 & $45.3 \pm 2.6$ & $37.1 \pm 2.4$ & $33.2 \pm 1.5$ & $31.3 \pm 2.6$ \\
\hline 54 & 13 & $41.4 \pm 2.5$ & $38.0 \pm 2.4$ & $28.8 \pm 1.4$ & $24.1 \pm 2.5$ \\
\hline 53 & 14 & $38.4 \pm 2.3$ & $35.5 \pm 2.3$ & $25.0 \pm 1.3$ & $19.8 \pm 2.3$ \\
\hline 52 & 15 & $43.4 \pm 2.4$ & $32.8 \pm 2.1$ & $22.9 \pm 1.2$ & $18.0 \pm 2.1$ \\
\hline 51 & 16 & $36.6 \pm 2.3$ & $32.1 \pm 2.2$ & $19.5 \pm 1.2$ & $13.3 \pm 2.1$ \\
\hline 50 & 17 & $33.4 \pm 2.1$ & $36.2 \pm 2.3$ & $18.3 \pm 1.1$ & $9.4 \pm 2.0$ \\
\hline 49 & 18 & $34.7 \pm 2.2$ & $30.7 \pm 2.1$ & $15.0 \pm 1.0$ & $7.1 \pm 1.8$ \\
\hline 48 & 19 & $29.5 \pm 2.1$ & $28.0 \pm 2.1$ & $14.1 \pm 1.0$ & $7.2 \pm 1.8$ \\
\hline 47 & 20 & $31.8 \pm 2.0$ & $27.1 \pm 2.0$ & $14.8 \pm 1.0$ & $8.7 \pm 1.8$ \\
\hline 46 & 21 & $34.2 \pm 2.1$ & $25.2 \pm 1.9$ & $11.5 \pm 0.9$ & $4.6 \pm 1.6$ \\
\hline 45 & 22 & $29.1 \pm 1.9$ & $27.9 \pm 2.0$ & $12.3 \pm 0.9$ & $4.5 \pm 1.7$ \\
\hline 44 & 23 & $32.4 \pm 2.0$ & $24.0 \pm 1.8$ & $12.1 \pm 0.9$ & $6.2 \pm 1.6$ \\
\hline 43 & 24 & $29.6 \pm 2.0$ & $26.8 \pm 1.9$ & $10.6 \pm 0.8$ & $2.5 \pm 1.6$ \\
\hline 42 & 25 & $26.7 \pm 1.8$ & $24.5 \pm 1.8$ & $10.9 \pm 0.9$ & $4.1 \pm 1.6$ \\
\hline 41 & 26 & $34.6 \pm 2.1$ & $25.5 \pm 1.8$ & $8.4 \pm 0.7$ & $-0.1 \pm 1.4$ \\
\hline 40 & 27 & $28.2 \pm 1.9$ & $25.5 \pm 1.9$ & $9.2 \pm 0.8$ & $1.0 \pm 1.5$ \\
\hline 39 & 28 & $24.9 \pm 1.7$ & $28.0 \pm 1.9$ & $8.9 \pm 0.7$ & $-0.6 \pm 1.5$ \\
\hline 38 & 29 & $28.4 \pm 1.8$ & $25.0 \pm 1.8$ & $9.1 \pm 0.7$ & $1.1 \pm 1.4$ \\
\hline
\end{tabular}

representative examples of such fits to the data. The only exception to this behavior is for the gold beam at large charge changes, Fig. 10, where the occurrence of fission causes a peak to appear in the $\sigma(\Delta Z)$ distributions, Sec. IV D. In addition, the values of $\sigma(\Delta Z)$ for

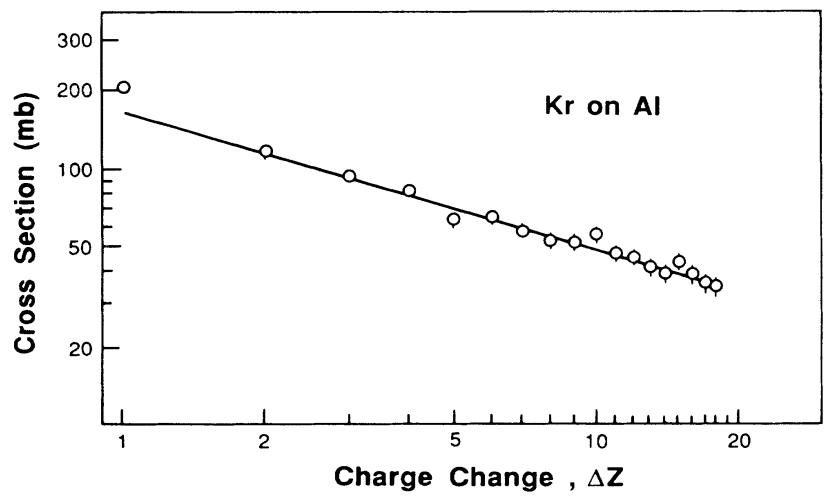

FIG. 7. The partial cross sections of krypton nuclei on an aluminum target as a function of $\Delta Z$, plotted on $\log$ - log scales. Also shown is the best fit for $2 \leq \Delta Z \leq 20$.
$\Delta Z=1$ nearly always appear to be anomalously high, with the excess cross-sections given in Table IX, due presumably to the additional process of electromagnetic dissociation causing proton stripping. These excess cross sections, while poorly defined due to the consider-

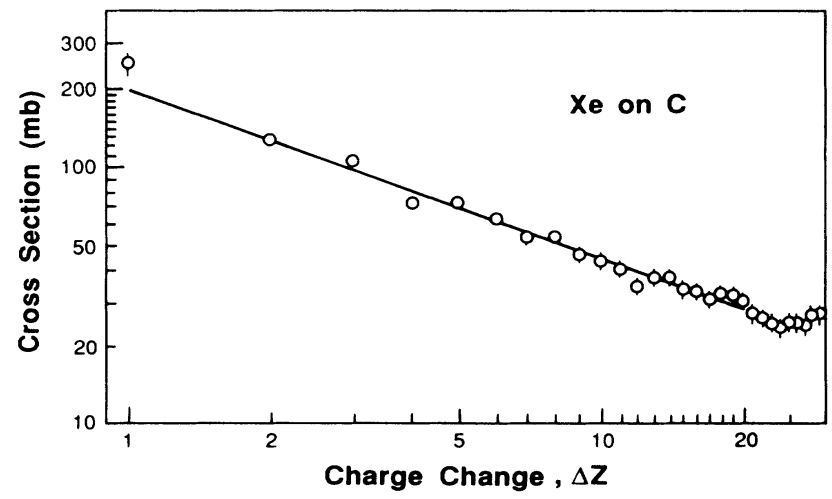

FIG. 8. The partial cross sections of xenon nuclei on a carbon target as a function of $\Delta Z$, plotted on log-linear scales. Also shown is the best fit for $2 \leq \Delta Z \leq 20$. 
TABLE VII. Cross sections of xenon in various media.

\begin{tabular}{lccccc}
\hline \hline $\boldsymbol{Z}$ & $\Delta \boldsymbol{Z}$ & $\begin{array}{c}\text { Aluminum } \\
(\mathrm{mb})\end{array}$ & $\begin{array}{c}\text { Carbon } \\
(\mathrm{mb})\end{array}$ & $\begin{array}{c}\text { Polyethylene } \\
(\mathrm{mb})\end{array}$ & $\begin{array}{c}\text { Hydrogen } \\
(\mathrm{mb})\end{array}$ \\
\hline 53 & 1 & $237.5 \pm 20.6$ & $249.3 \pm 21.3$ & $230.0 \pm 9.1$ & $220.3 \pm 17.3$ \\
52 & 2 & $133.6 \pm 7.2$ & $127.3 \pm 4.9$ & $136.4 \pm 3.5$ & $141.0 \pm 5.9$ \\
51 & 3 & $95.7 \pm 6.0$ & $104.9 \pm 4.3$ & $104.4 \pm 3.0$ & $104.1 \pm 5.0$ \\
50 & 4 & $76.8 \pm 5.3$ & $71.9 \pm 3.6$ & $94.2 \pm 2.8$ & $105.3 \pm 4.6$ \\
49 & 5 & $68.1 \pm 5.1$ & $72.6 \pm 3.6$ & $78.7 \pm 2.6$ & $81.7 \pm 4.3$ \\
48 & 6 & $65.7 \pm 4.8$ & $63.0 \pm 3.3$ & $72.6 \pm 2.4$ & $77.4 \pm 4.0$ \\
47 & 7 & $56.8 \pm 4.4$ & $53.0 \pm 2.9$ & $65.3 \pm 2.2$ & $71.5 \pm 3.6$ \\
46 & 8 & $51.5 \pm 4.3$ & $53.3 \pm 3.0$ & $53.4 \pm 2.0$ & $53.4 \pm 3.4$ \\
45 & 9 & $54.1 \pm 4.3$ & $45.3 \pm 2.7$ & $47.1 \pm 1.8$ & $48.0 \pm 3.1$ \\
44 & 10 & $51.8 \pm 4.2$ & $42.6 \pm 2.6$ & $43.1 \pm 1.8$ & $43.3 \pm 3.0$ \\
43 & 11 & $54.3 \pm 4.2$ & $40.2 \pm 2.5$ & $36.8 \pm 1.6$ & $35.2 \pm 2.7$ \\
42 & 12 & $50.4 \pm 4.2$ & $34.6 \pm 2.4$ & $32.7 \pm 1.6$ & $31.8 \pm 2.6$ \\
41 & 13 & $41.7 \pm 3.7$ & $37.3 \pm 2.4$ & $31.0 \pm 1.5$ & $27.9 \pm 2.6$ \\
40 & 14 & $39.2 \pm 3.6$ & $37.0 \pm 2.4$ & $27.5 \pm 1.4$ & $22.7 \pm 2.4$ \\
39 & 15 & $34.8 \pm 3.5$ & $33.3 \pm 2.3$ & $20.5 \pm 1.3$ & $14.1 \pm 2.2$ \\
38 & 16 & $38.6 \pm 3.5$ & $32.8 \pm 2.2$ & $18.6 \pm 1.2$ & $11.5 \pm 2.1$ \\
37 & 17 & $37.5 \pm 3.4$ & $30.5 \pm 2.2$ & $17.0 \pm 1.1$ & $10.3 \pm 2.0$ \\
36 & 18 & $35.5 \pm 3.3$ & $32.0 \pm 2.2$ & $16.0 \pm 1.1$ & $8.0 \pm 1.9$ \\
35 & 19 & $30.3 \pm 3.1$ & $31.6 \pm 2.2$ & $14.7 \pm 1.1$ & $6.3 \pm 1.9$ \\
34 & 20 & $29.2 \pm 3.0$ & $30.0 \pm 2.1$ & $13.8 \pm 1.0$ & $5.6 \pm 1.8$ \\
33 & 21 & $24.6 \pm 2.7$ & $26.9 \pm 2.0$ & $12.8 \pm 1.0$ & $5.8 \pm 1.8$ \\
32 & 22 & $32.6 \pm 3.2$ & $25.8 \pm 1.9$ & $12.9 \pm 1.0$ & $6.5 \pm 1.7$ \\
31 & 23 & $26.6 \pm 2.8$ & $24.8 \pm 1.9$ & $10.3 \pm 0.9$ & $3.1 \pm 1.6$ \\
30 & 24 & $27.2 \pm 3.0$ & $23.8 \pm 1.9$ & $11.9 \pm 0.9$ & $5.9 \pm 1.7$ \\
29 & 25 & $28.9 \pm 3.0$ & $25.0 \pm 1.9$ & $11.4 \pm 0.9$ & $0.8 \pm 1.6$ \\
28 & 26 & $40.4 \pm 3.5$ & $25.1 \pm 1.9$ & $10.7 \pm 0.9$ & $4.5 \pm 1.6$ \\
27 & 27 & $36.7 \pm 3.3$ & $24.4 \pm 1.9$ & $26.7 \pm 2.0$ & $3.9 \pm 1.6$ \\
26 & 28 & $36.2 \pm 3.3$ & $27.1 \pm 1.9$ & & $2.0 \pm 1.6$ \\
25 & 29 & $33.1 \pm 3.1$ & & & $-9.9 \pm 1.5$ \\
\hline \hline
\end{tabular}

able errors, are in reasonable agreement with those reported by Mercier et al. ${ }^{7}$ for one neutron removal from heavy targets by light projectiles.

For the targets with heavy nuclei, aluminum, and carbon, the variation in the cross sections, for $2 \leq \Delta Z \leq 20$,

TABLE VIII. Cross sections of krypton.

\begin{tabular}{lcc}
\hline$Z$ & $\Delta Z$ & Aluminum $(\mathrm{mb})$ \\
\hline 35 & 1 & $204.27 \pm 6.15$ \\
34 & 2 & $114.16 \pm 5.2$ \\
33 & 3 & $92.74 \pm 4.38$ \\
32 & 4 & $80.74 \pm 4.11$ \\
31 & 5 & $62.62 \pm 3.47$ \\
30 & 6 & $64.45 \pm 3.69$ \\
29 & 7 & $56.94 \pm 3.45$ \\
28 & 8 & $51.63 \pm 3.17$ \\
27 & 9 & $51.37 \pm 3.08$ \\
26 & 10 & $54.47 \pm 3.28$ \\
25 & 11 & $46.1 \pm 2.91$ \\
24 & 12 & $44.33 \pm 2.76$ \\
23 & 13 & $40.76 \pm 2.69$ \\
22 & 14 & $38.28 \pm 2.69$ \\
21 & 15 & $42.61 \pm 2.74$ \\
20 & 16 & $38.33 \pm 2.63$ \\
19 & 17 & $35.25 \pm 2.52$ \\
18 & 18 & $34.31 \pm 2.44$ \\
\hline \hline
\end{tabular}

can be fitted with acceptable values of reduced $\chi^{2}$, Table IV, by simple power law expressions of the form

$$
\sigma(\Delta Z)=\Sigma_{0}(\Delta Z)^{-\alpha} \mathrm{mb}
$$

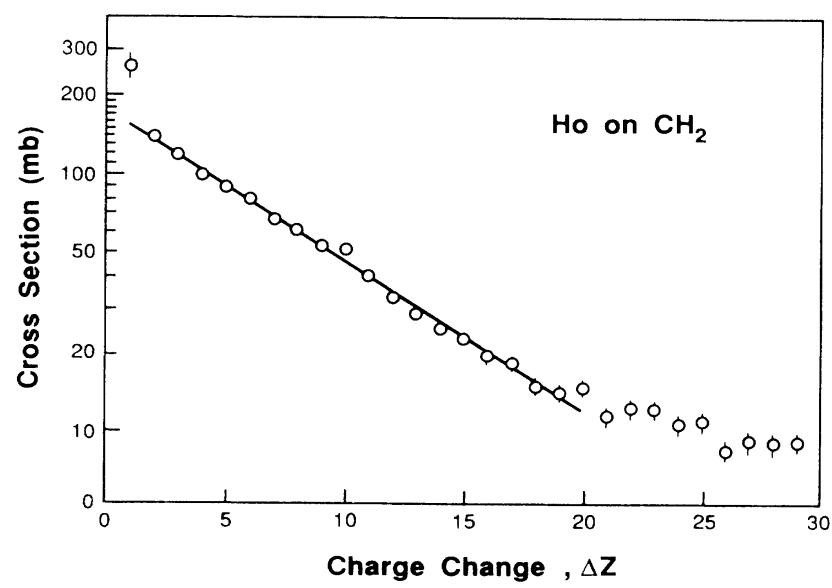

FIG. 9. The partial cross sections of holmium nuclei on a polyethylene target as a function of $\Delta Z$, plotted on log-log scales. Also shown is the best fit for $2 \leq \Delta Z \leq 20$. 


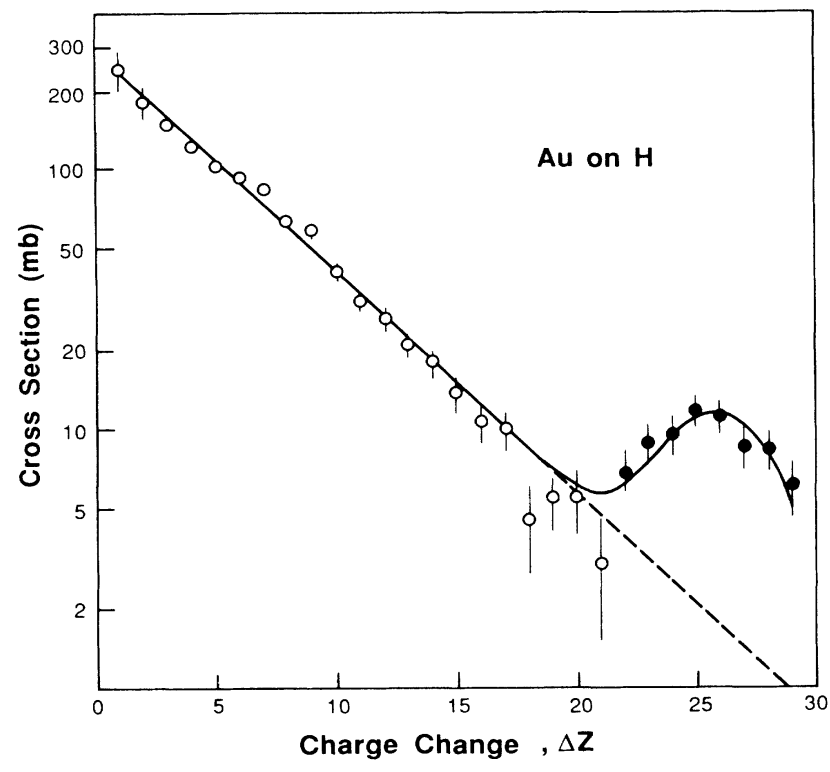

FIG. 10. The partial cross sections of gold nuclei on a hydrogen target as a function of $\Delta Z$, plotted on log-linear scales, showing the fission peak at high $\Delta Z$. The values that have an appreciable fission component are shown by solid points. Also, the exponential fit has been extended into the fission region as a dotted line.

where $\Sigma_{0}\left(Z_{B}, Z_{T}\right)$ and $\alpha\left(Z_{B}, Z_{T}\right)$ are constants for each beam and target.

For the targets which contain hydrogen, i.e., polyethylene $\left(\mathrm{CH}_{2}\right)$ and hydrogen, the cross sections are not well fitted by this power law form, e.g., for holmium nuclei on a hydrogen target an attempted power law fit has a reduced $\chi^{2}$ of 26 , but instead can be reasonably well represented by exponential expressions of the form

$$
\sigma(\Delta Z)=\sigma_{0} \exp \left[-(\Delta Z) / \Delta_{0}\right] \mathrm{mb}
$$

where $\sigma_{0}\left(Z_{B}, Z_{T}\right)$ and $\Delta_{0}\left(Z_{B}, Z_{T}\right)$ are constants for each beam and target. Values of these fitting parameters, $\Sigma_{0}$, $\alpha, \sigma_{0}$, and $\Delta_{0}$, determined over the range $2 \leq \Delta Z \leq 20$, are given in Table IV, together with values of reduced $\chi^{2}$. It can be seen that these simple fits are remarkably good representations of the data over a wide range of $\Delta Z$. However, it is clear that they cannot cover the entire range of $\Delta Z$ since $\sigma(\Delta Z)$ summed over all $\Delta Z$ must equal $\sigma_{\text {tot }}$. Although neither Eqs. (12) or (13) can be in-

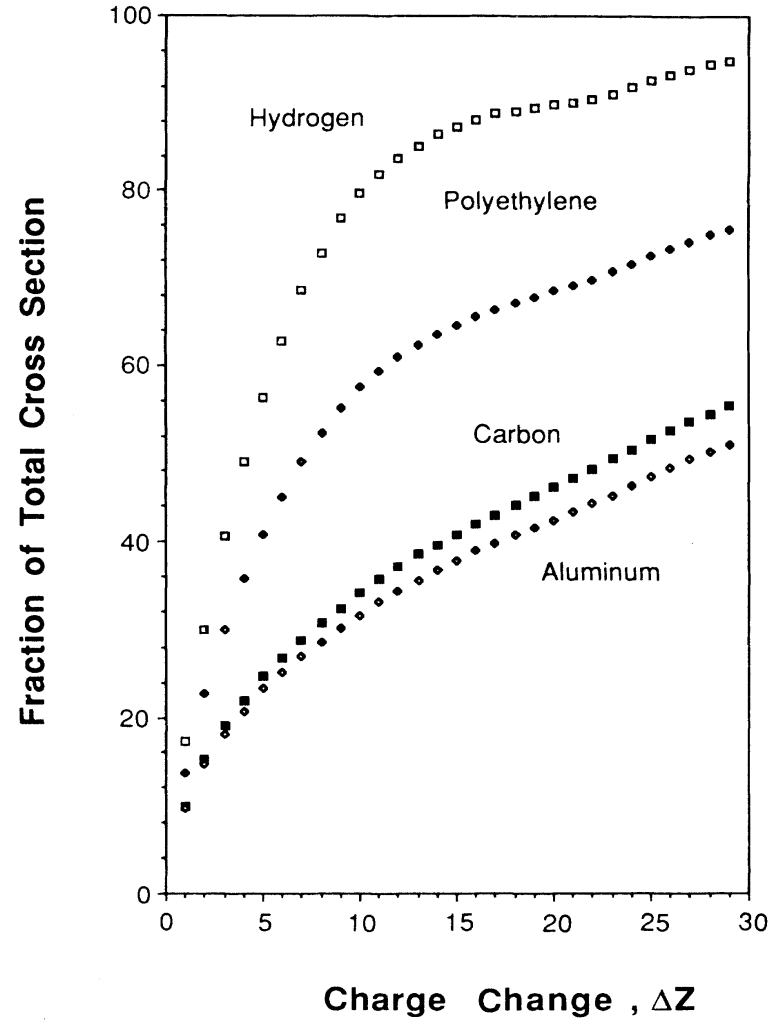

FIG. 11. The incremental sums of the partial cross sections listed in Table $\mathrm{V}$ as a function of the charge change, for gold nuclei on all targets.

tegrated, due to their discrete nature, summation of the individual values show that quite appreciable fractions of $\sigma_{\text {tot }}$ are reached even when only summing up to $\Delta Z=20$. For example, Fig. 11 shows the fraction of the total cross sections represented by these sums for gold nuclei on all targets. This figure shows that, as might be expected, the lighter the target the greater the probability of producing a fragment with a small $\Delta Z$. It also shows that the partial cross sections directly measured in this experiment, for $\Delta Z<30$, account for as much as $95 \%$ of the total cross section for gold on hydrogen, but only for about half of the total cross section for gold on carbon or aluminum.

The values of the reduced $\chi^{2}$ found for the exponential fits, Table IV, show that they are not as good as would be obtained from a more complex expression. However Eq. (13) does provide a reasonable representation and organization of the data. Such an exponential relation

TABLE IX. Excess cross sections for $\Delta Z=1$ fragments, in mb.

\begin{tabular}{lcccc}
\hline & & \multicolumn{2}{c}{ Target nuclei } & \\
Beam nuclei & Hydrogen & Polyethylene & Carbon & Aluminum \\
\hline gold & $30 \pm 41$ & $68 \pm 26$ & $46 \pm 27$ & $49 \pm 31$ \\
holmium & $97 \pm 42$ & $112 \pm 26$ & $57 \pm 28$ & $19 \pm 27$ \\
xenon & $67 \pm 17$ & $85 \pm 9$ & $42 \pm 21$ & $52 \pm 21$ \\
krypton & & & $41 \pm 6$ \\
\hline \hline
\end{tabular}


would be expected based on the semiempirical fits to measurements of cross sections of individual isotopes produced from the spallation of heavy nuclei by proton bombardment, such as that reported by Rudstam ${ }^{8}$ and originally applied to the calculation of elemental cross sections by Waddington. ${ }^{9}$ The later extensions by Silberberg and Tsao $^{1}$ still maintain the basic exponential nature of Eq. (13).

\section{Charge and energy dependence}

It is known from work with lighter beams by Webber ${ }^{10}$ that there are significant energy dependences in the individual partial cross sections. Webber's results with incident iron nuclei can be used to determine values for the fitting parameters in hydrogen, polyethylene and carbon targets, just as in our analysis for the heavier beams. Figure 12 shows, for example, the variation in $\sigma_{0}(26,1)$ and $\Delta_{0}(26,1)$ with energy, deduced from the cross sections for iron nuclei on hydrogen. It can be
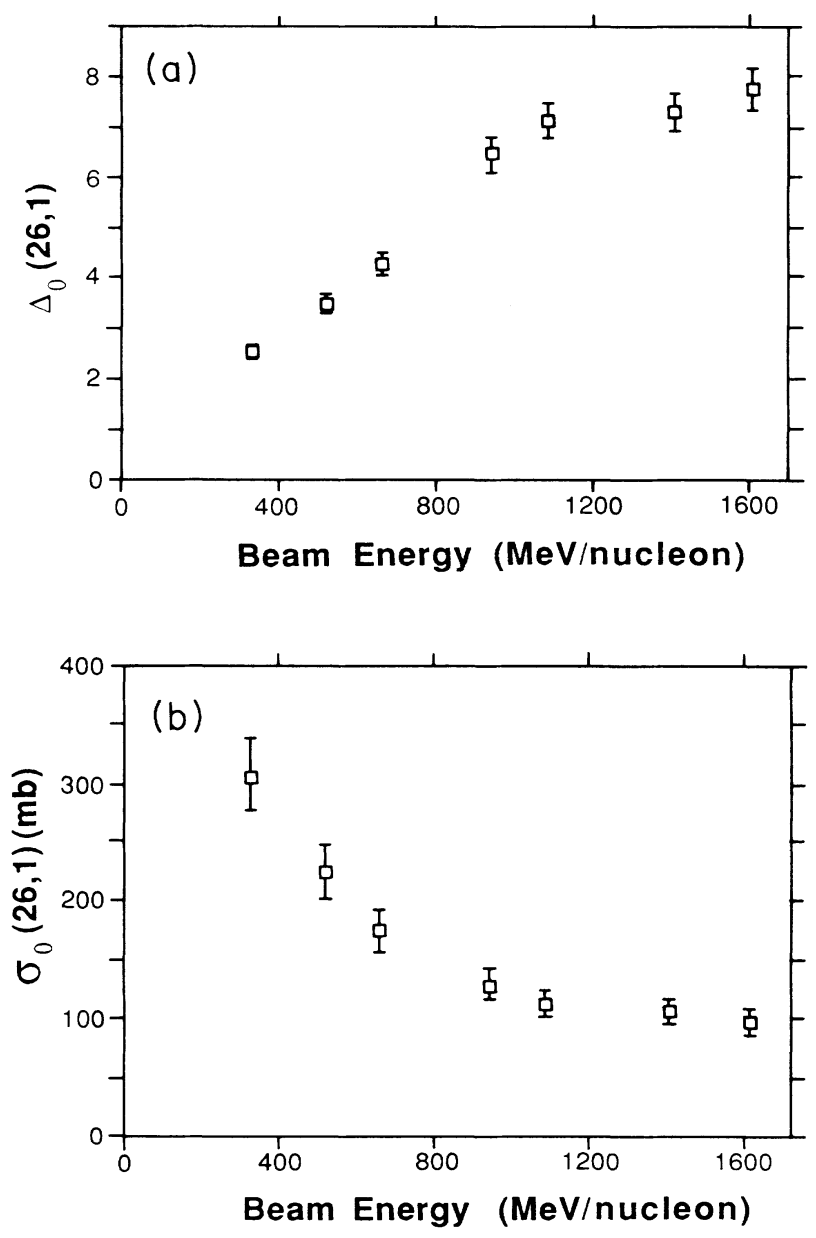

FIG. 12. (a) The energy dependence of the fitting parameter $\Delta_{0}$ derived from the data of Webber (Ref. 10) for iron nuclei on a hydrogen target. (b) The energy dependence of the fitting parameter $\sigma_{0}$ derived from the data of Webber (Ref. 10) for iron nuclei on a hydrogen target. seen that for these relatively light projectile nuclei there is little evidence of any energy variation above about 1.0 $\mathrm{GeV} /$ nucleon, but considerable variation at lower energies.

However, studies by Kaufman and Steinberg ${ }^{11}$ of the
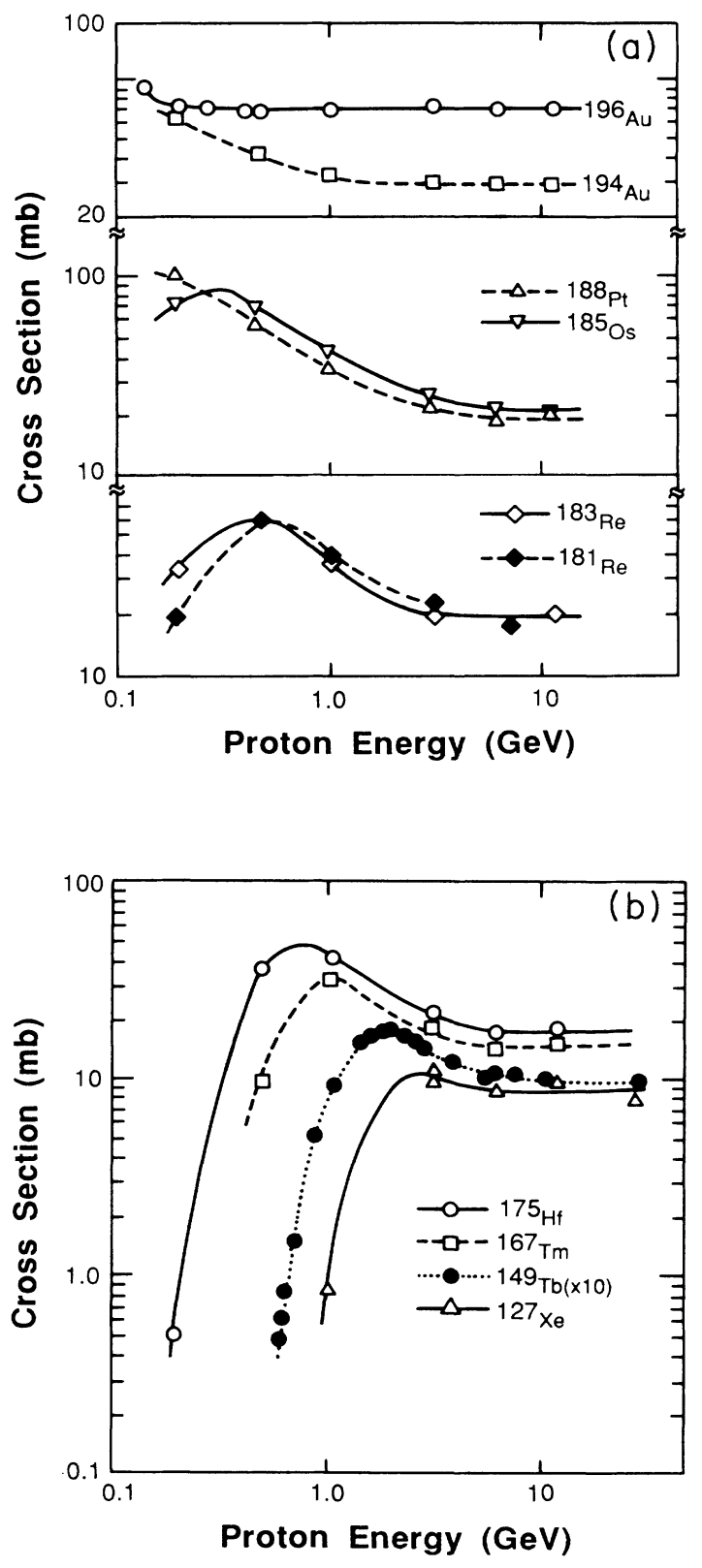

FIG. 13. (a) The variation with energy of the partial cross sections for several isotopes with small mass changes produced by the bombardment of gold nuclei with protons of different energy [from Kaufman and Steinberg (Ref. 11)]. (b) The variation with energy of the partial cross sections for several isotopes with larger mass changes produced by the bombardment of gold nuclei with protons of different energy [from Kaufman and Steinberg (Ref. 11)]. 
partial cross sections for the production of individual unstable isotopes by proton bombardment of various targets much heavier than iron have shown considerable energy dependence extending significantly higher than 1 $\mathrm{GeV} /$ nucleon. Examples of this are shown in Fig. 13, which illustrates the energy dependence of the cross sections for several isotopes of small $\Delta Z$, Fig. 13(a), and large $\Delta Z$, Fig. 13(b), produced by the bombardment of a gold target with protons of various energies. It can be seen that these cross sections vary with energy up to much higher energies than any available at the Bevalac. Clearly we have to expect to observe energy dependent changes in our data, although they are unlikely to be large due to the rather small range of energy that we covered.

We begin our discussion of the dependence of the fitting parameters on charge and energy by considering the aluminum target data. Figures $14(\mathrm{a})$ and $14(\mathrm{~b})$ shows $\Sigma_{0}\left(Z_{B}, Z_{T}\right)$ and $\alpha\left(Z_{B}, Z_{T}\right)$ plotted against $Z_{B}$ for the aluminum targets, displaying a linear dependence on charge for both parameters. Unfortunately, in this experiment $Z_{B}$ was also correlated with the beam energy, $E_{B}$, since each beam was accelerated to its unique maximum attainable energy. Figures $14(\mathrm{c})$ and 14(d) show the same values of $\Sigma_{0}\left(Z_{B}, Z_{T}\right)$ and $\alpha\left(Z_{B}, Z_{T}\right)$ for the aluminum targets plotted against $E_{B}$ rather than $Z_{B}$. Here again the data show a dependence on the plotted variable, in this case the energy, but with a suggestion that the values of both parameters are becoming less dependent on energy at the highest energies. Thus in the case of the aluminum targets we cannot distinguish between the charge and energy dependence.

Fortunately, for the other targets we are able to address this question in a different manner, because we also have available Webber's data for a beam of iron nuclei over a range of energies. Figure $15(\mathrm{a})$ shows $\Sigma_{0}\left(Z_{B}, Z_{T}\right)$ as a function of energy for the carbon targets, both for our results at their individual energies, and for Webber's
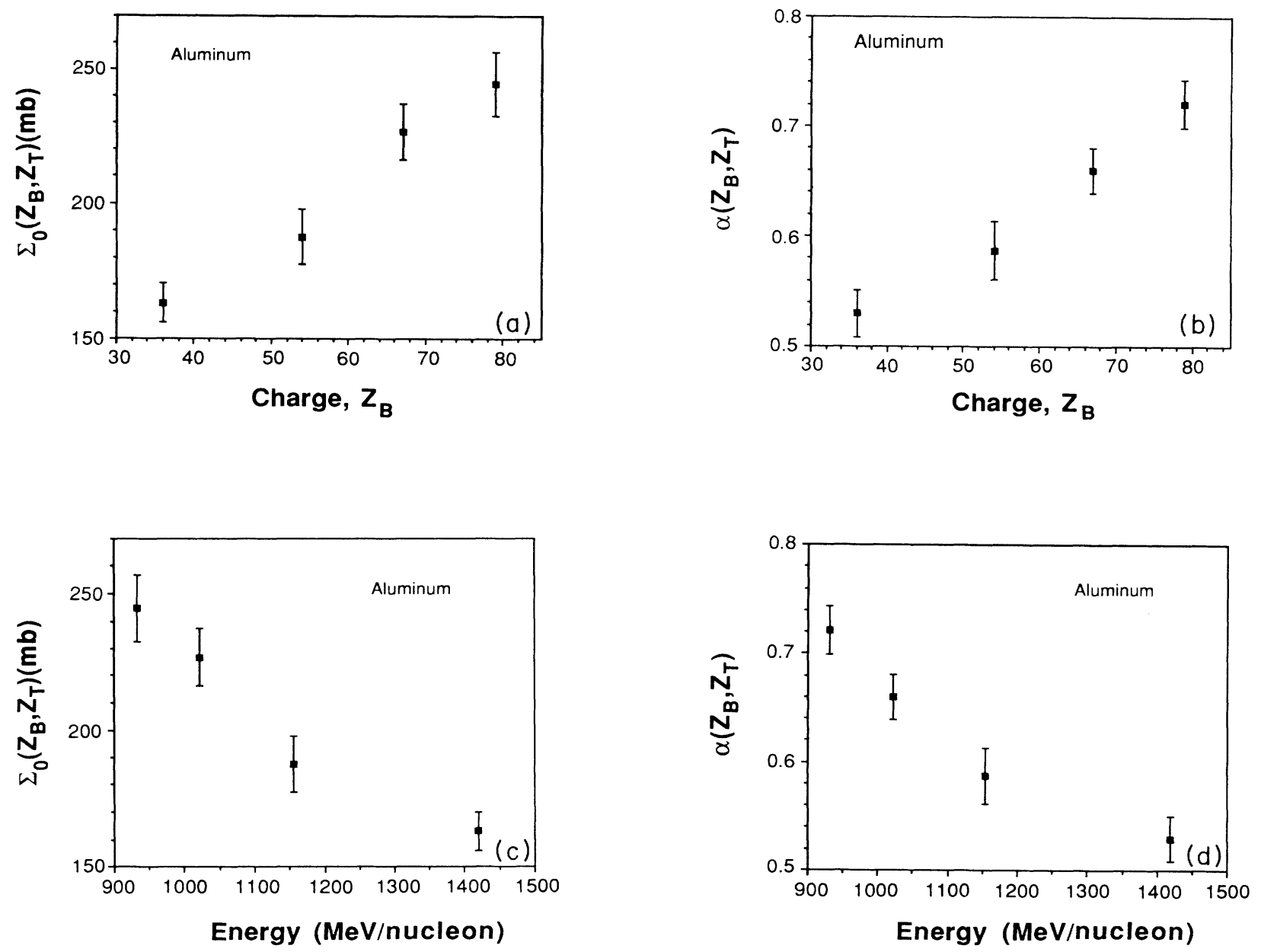

FIG. 14. (a) Fitting parameter $\Sigma_{0}\left(Z_{B}, Z_{T}\right)$, in mb, as a function of beam charge, $Z_{B}$, for aluminum targets. (b) Fitting parameter $\alpha\left(Z_{B}, Z_{T}\right)$ as a function of beam charge, $Z_{B}$, for aluminum targets. (c) Fitting parameter $\Sigma_{0}\left(Z_{B}, Z_{T}\right)$, in mb, as a function of beam energy, $E_{B}$, for aluminum targets. (d) Fitting parameter $\alpha\left(Z_{B}, Z_{T}\right)$ as a function of beam energy, $E_{B}$, for aluminum targets. 
iron nuclei at several energies directly comparable with those of our data. The differences in $\Sigma_{0}\left(Z_{B}, Z_{T}\right)$ for two projectiles at the same energy then represent the charge dependence of $\Sigma_{0}\left(Z_{B}, Z_{T}\right)$ at that particular energy. In Fig. 15(a) these are the vertical displacements between our measured values and the points shown as open circles on the line connecting the iron data points. An alternative way of showing the same result is to plot
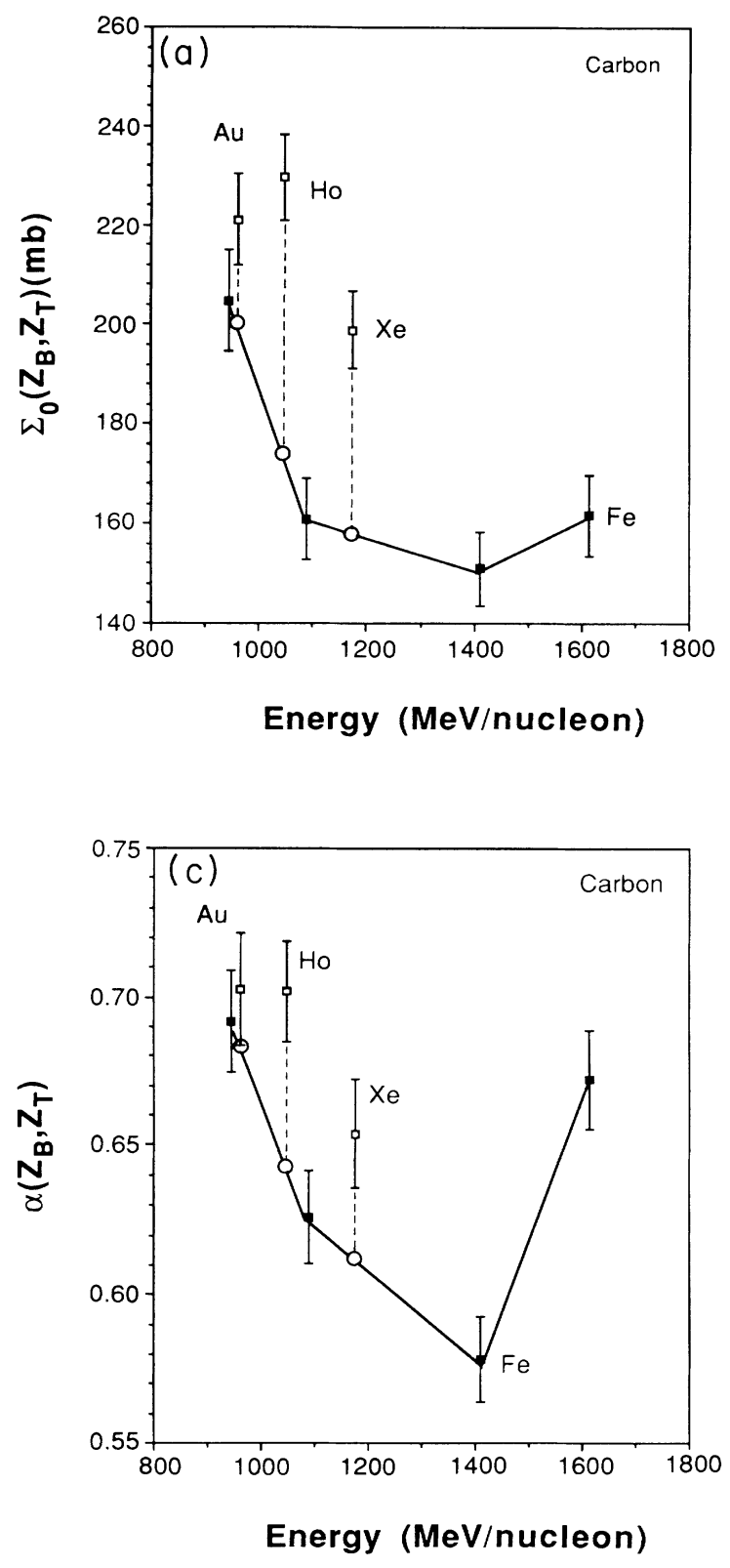

$\Sigma_{0}\left(Z_{B}, Z_{T}\right)$ as a function of the charge, and then to connect each of our values to the interpolated iron value at the same energy, see Fig. 15(b). The slopes of these lines can be taken to represent the charge dependence of $\Sigma_{0}\left(Z_{B}, Z_{T}\right)$ at a particular energy, while the spread between the different lines represents the energy dependence. Figures $15(\mathrm{c})$ and $15(\mathrm{~d})$ display $\alpha\left(Z_{B}, Z_{T}\right)$ in the same fashion.
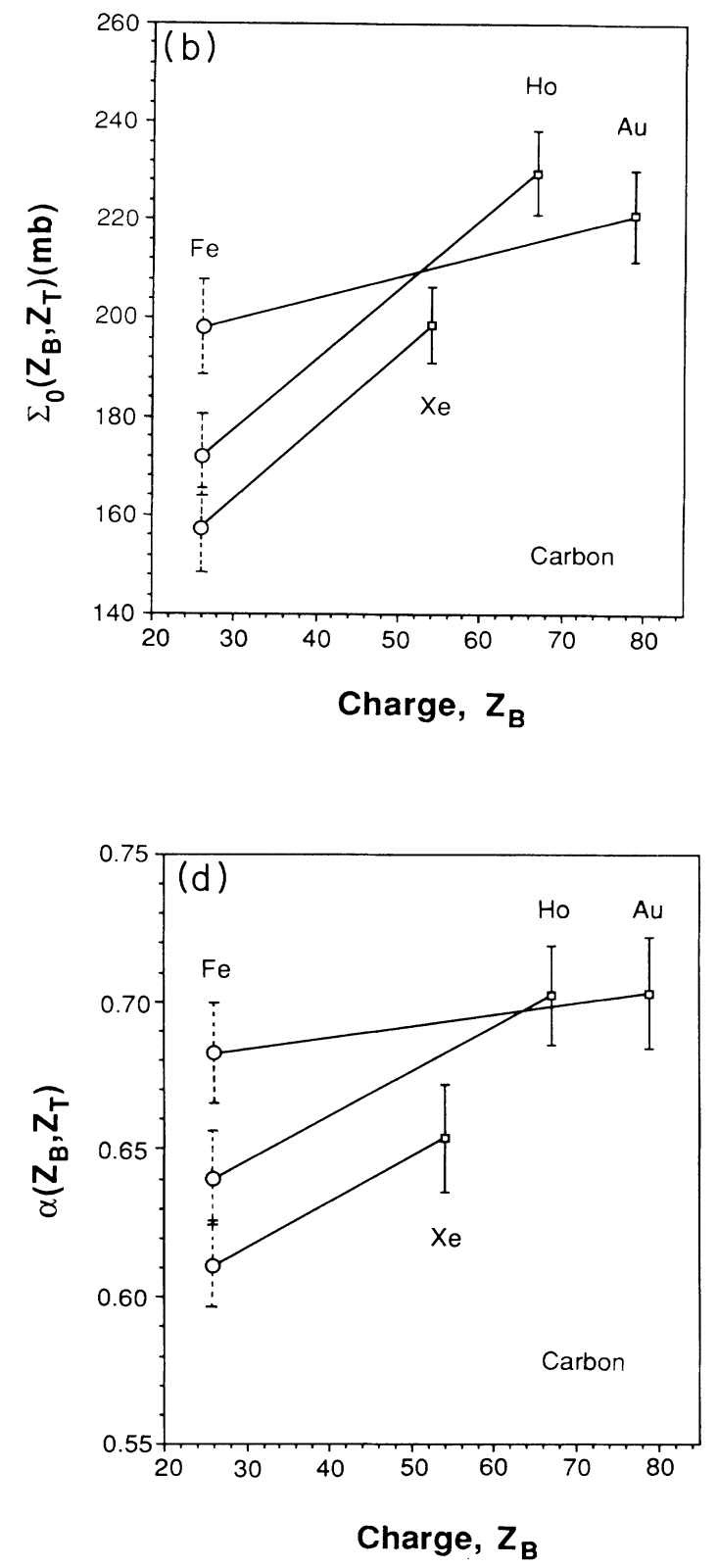

FIG. 15. (a) Fitting parameter $\Sigma_{0}\left(Z_{B}, Z_{T}\right)$, in mb, as a function of beam energy, $E_{B}$, for carbon targets. Points marked iron are derived from Webber's data (Ref. 10) and are connected to guide the eye. (b) Fitting parameter $\Sigma_{0}\left(Z_{B}, Z_{T}\right)$, in mb, as a function of beam charge, $Z_{B}$, for carbon targets. Each value for our data is connected to an iron value of the same energy, with an error similar to those on the iron data points, as deduced from (a). (c) Fitting parameter $\alpha\left(Z_{B}, Z_{T}\right)$ as a function of beam energy, $E_{B}$, for carbon targets. Points marked iron are derived from Webber's data (Ref. 10) and are connected to guide the eye. (d) Fitting parameter $\alpha\left(Z_{B}, Z_{T}\right)$ as a function of beam charge, $Z_{B}$, for carbon targets. Each value for our data is connected to an iron value of the same energy, with an error similar to those on the iron data points, as deduced from (c). 
The fitting parameters for the hydrogen and polyethylene targets can be examined in the same manner as for the carbon targets. Figures 16 and 17 give values of the fitting parameters $\sigma_{0}\left(Z_{B}, Z_{T}\right)$ and $\Delta_{0}\left(Z_{B}, Z_{T}\right)$ as a function of energy and charge. It can be seen that although the parameters are not as well organized as those for the carbon targets, there is clear evidence for a charge dependence in each case, and also evidence for some energy dependence, even over the small range of energy covered by our results. We cannot distinguish
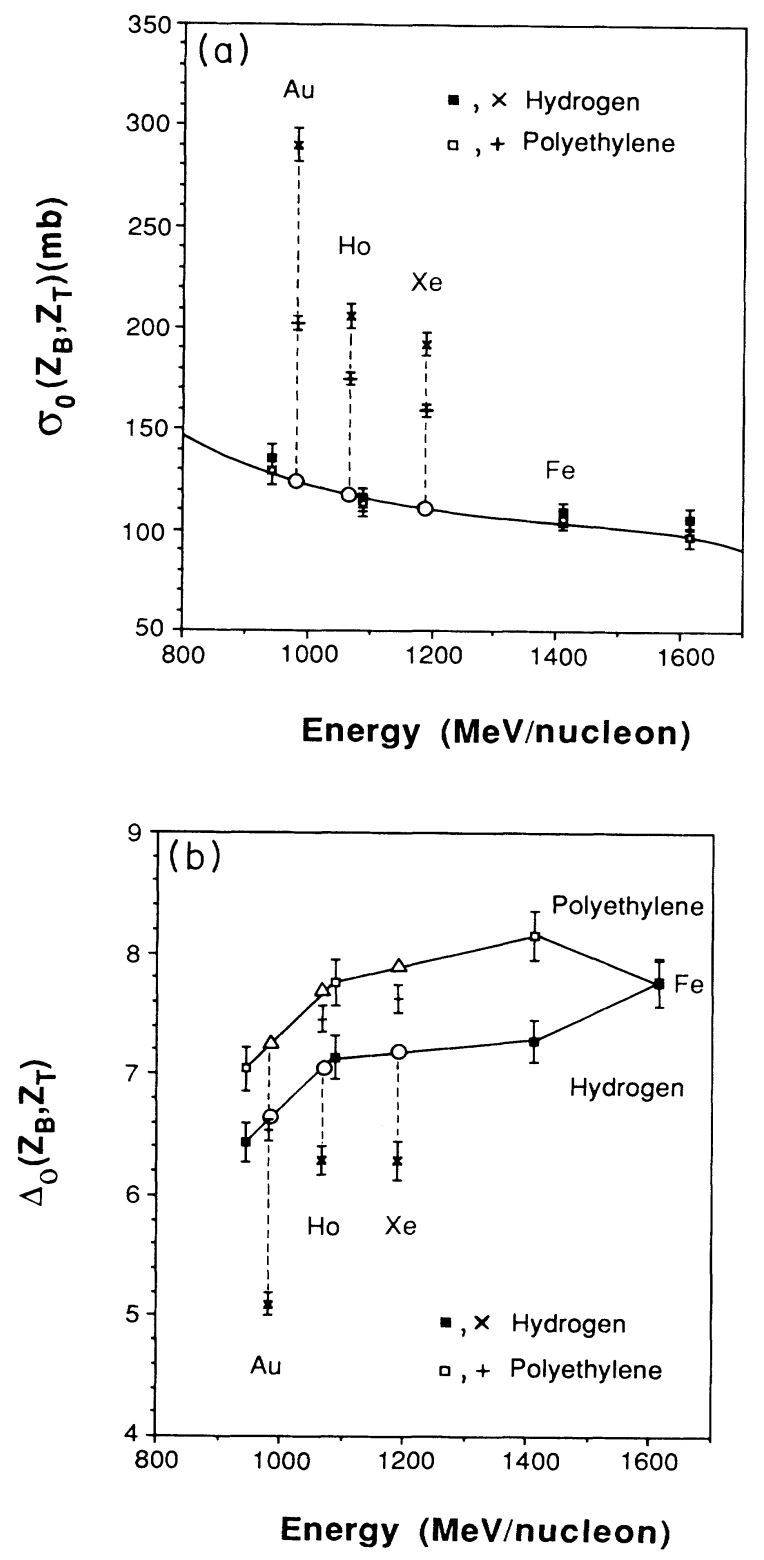

FIG. 16. (a) Fitting parameter $\sigma_{0}\left(Z_{B}, Z_{T}\right)$, in $\mathrm{mb}$, as a function of beam energy, $E_{B}$, for hydrogen and polyethylene targets. Points marked iron are derived from Webber's data (Ref. 10) and are connected to guide the eye. (b) Fitting parameter $\Delta_{0}\left(Z_{B}, Z_{T}\right)$ as a function of beam energy, $E_{B}$, for hydrogen and polyethylene targets. Points marked iron are derived from Webber's data (Ref. 10) and are connected to guide the eye. between these charge and energy dependences of the cross-sections, and hence we cannot reliably predict the behavior of the cross sections at other charges or other energies, particularly when we also consider the evidence from the $\mathrm{p}$ - $A$ data $^{10}$ of energy dependence over a wide range of energy. At best, from our own data, we could assume that the entire variation observed is due to a dependence on only the charge, and use this assumption to derive "asymptotic" values. Previously this is the as-

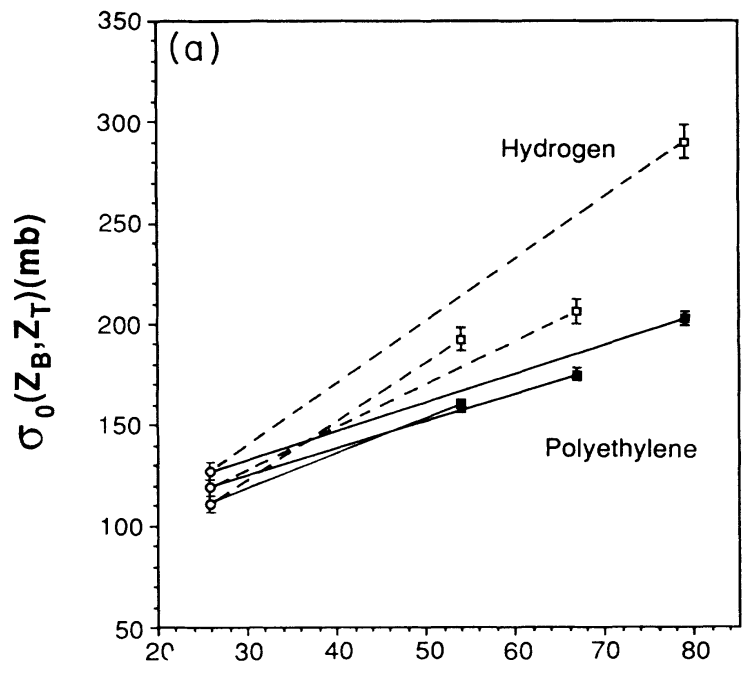

Charge, $Z_{B}$

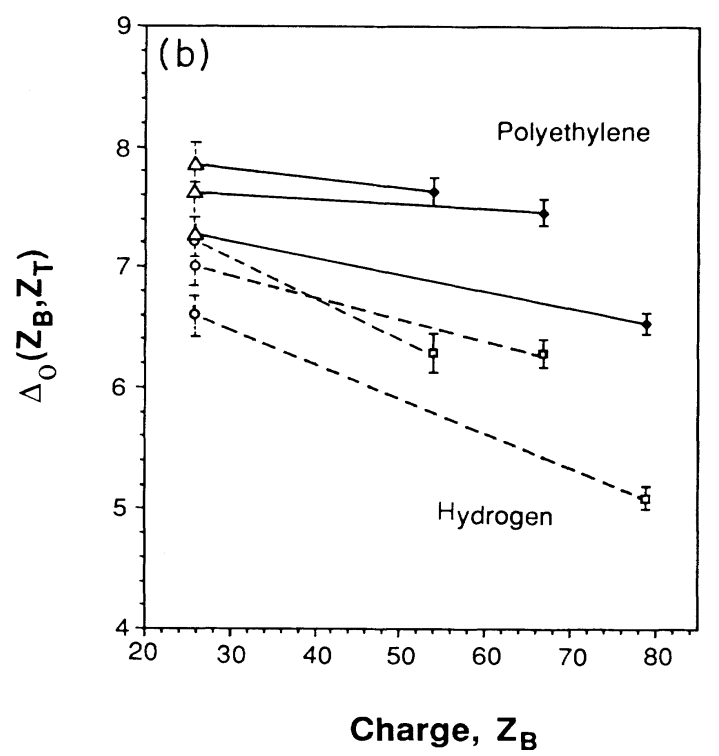

FIG. 17. (a) Fitting parameter $\sigma_{0}\left(Z_{B}, Z_{T}\right)$, in mb, as a function of beam energy, $E_{B}$, for hydrogen and polyethylene targets. Each value for our data is connected to an iron value of the same energy, with an error similar to those on the iron data points, as deduced from Fig. 16(a). (b) Fitting parameter $\Delta_{0}\left(Z_{B}, Z_{T}\right)$ as a function of beam charge, $Z_{B}$, for hydrogen and polyethylene targets. Each value for our data is connected to an iron value of the same energy, with an error similar to those on the iron data points, as deduced from Fig. 16(b). 


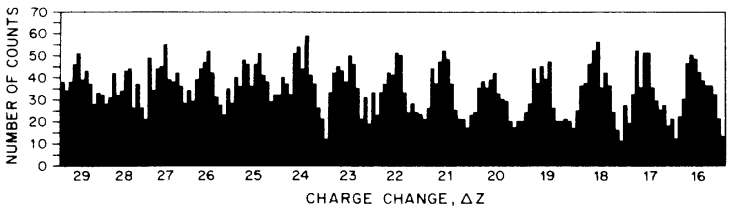

FIG. 18. Charge histogram in the fission region for gold nuclei on a polyethylene target.

sumption that has been made in our cosmic ray propagation studies and applied to the HEAO data by Binns et al. ${ }^{12}$ and Brewster et al., ${ }^{13}$ where we assumed that the cross-sections were energy independent. From our discussion here, and from the $\mathrm{p}-A$ data, ${ }^{10}$ we have to conclude that this assumption can probably no longer be justified, although it should not significantly affect our major conclusions, since the majority of the nuclei in the HEAO data set are of quite high energy where the cross sections should have reached asymptotic values, which, although not necessarily the ones we assumed, should not show major systematic differences.

\section{Fission cross sections}

While the majority of the cross sections show a steady decrease with increasing charge change, the gold cross sections, e.g., see Fig. 10, show a clear rise to a peak at a $\Delta Z$ of about 25 beginning at a $\Delta Z$ of about 20 . This peak can be attributed to the fission of energetic gold nuclei which would result in the production of two heavy nuclei whose summed charges would be close to that of the primary gold nucleus, giving a signal in our detectors corresponding to that of a single particle with an apparent charge of between 50 and 55 , or a $\Delta Z$ of between 29 and 24 . The observation of individual charge peaks in this region, Fig. 18, shows that many of the observed events are still due to single fragments but that there is an underlying background of fission events which produce the elevated cross sections. As a consequence, the values derived for $\sigma(\Delta Z)$ in this charge region do not represent just the production cross sections for single fragments, but include a variable amount due to the fission background.

To estimate the fission cross section it was assumed that the trend in $\sigma(\Delta Z)$ observed at smaller charge changes extends into the region of the fission reactions, as shown by the dashed line in Fig. 10. The measured cross sections in the fission region were then fit to the appropriate expression, Eq. (12) or Eq. (13), plus a Gaussian function to represent the total fission cross section. The area under the Gaussian is then taken as the integrated fission cross section. The fission cross section for a hydrogen target deduced in this experiment of $63 \pm 9 \mathrm{mb}$, or $4.4 \%$ of the total cross section, is in good agreement with that of $71 \pm 7 \mathrm{mb}$ observed by Vaishene et al. ${ }^{14}$ who bombarded gold with $1.0 \mathrm{GeV}$ protons. The fission cross sections in the other targets, polyethylene, carbon, and aluminum, were, respectively, $57 \pm 6,46 \pm 5$, and $64 \pm 7 \mathrm{mb}$, and thus appear to be relatively independent of the target charge, at least for these light targets. These values can be compared with those observed in nuclear emulsion, which contains much heavier target nuclei. For incident $1.0 \mathrm{GeV} /$ nucleon gold nuclei, which slow down and stop in the emulsions, Waddington and Freier ${ }^{15}$ found that for "pure" fission, with no accompanying protons or alpha particles, the fission cross section was $44 \pm 15 \mathrm{mb}$ while for "dirty" fission, with up to five accompanying charges, it was $147 \pm 27 \mathrm{mb}$. It should be noted that the analysis procedure we have used here assumes the validity of the fitting expressions, Eqs. (12) and (13), in a region of $\Delta Z$ beyond that for which they were derived.

\section{CONCLUSIONS}

We have measured total and partial charge changing cross sections for various heavy projectile nuclei incident on various light targets at energies between 0.9 and 1.4 $\mathrm{GeV} /$ nucleon. The total cross sections fit a geometrical overlap model with the overlap depending on the sum of the masses of the target and the projectile nuclei. The partial cross sections for a given target and projectile fit a simple power law for carbon and aluminum targets and a simple exponential for polyethylene and hydrogen targets. The parameters which characterize these power laws and exponentials appear to be dependent on both the charge and energy of the projectile, but this set of measurements is inadequate to separate these two effects. Subsequent measurements, made over a range of energies, should enable us to differentiate the charge and energy effects, at least over the range of energies available from the Bevalac.

\section{ACKNOWLEDGMENTS}

An experiment of this magnitude can only be carried to a successful conclusion with the help of many people. We are indebted to W. E. Althouse, J. A. Becker, J. W. Epstein, B. W. Gauld, G. Grimm, and B. J. Newport from our own groups. The personnel at Lawrence Berkeley Laboratory made the runs possible and we particularly wish to thank H. J. Crawford, I. Flores, and P. J. Lindstrom for their help. W. R. Webber provided us with his data on the iron interactions prior to publication. This work was funded in part by NASA under Grants Nos. NAG-8-498, 8-500, 8-502, and NGR 05002-160, 24-005-050, and 26-008-001.
${ }^{1}$ R. Silberberg and C. H. Tsao, Ap. J. Suppl. 25, 315 (1973); 25, 335 (1973). See also R. Silberberg, C. H. Tsao, and J. R. Letaw, in Composition and Origin of Cosmic Rays, edited by M. M. Shapiro (Reidel, Dordrecht, 1983), p. 321.
${ }^{2}$ W. R. Binns, M. H. Israel, J. Klarmann, W. R. Scarlett, E. C. Stone, and C. J. Waddington, Nucl. Instr. Methods 185, 415 (1981).

${ }^{3}$ B. J. Newport, E. C. Stone, C. J. Waddington, W. R. Binns, 
D. J. Fixsen, T. L. Garrard, G. Grimm, M. H. Israel, and J. Klarmann, Proceedings of the 19th International Conference on Cosmic Rays, NASA Conference Publication 2376, 1985, Vol. 3, p. 287.

${ }^{4}$ M. P. Kertzman, Ph.D. thesis, University of Minnesota, 1987.

${ }^{5}$ G. D. Westfall, L. W. Wilson, P. J. Lindstrom, H. J. Crawford, D. E. Greiner, and H. H. Heckman, Phys. Rev. C 19, 1309 (1979).

${ }^{6} \mathrm{~F}$. Hagen, Ph.D. thesis, University of Maryland, 1976.

${ }^{7}$ M. T. Mercier, J. C. Hill, F. K. Wohn, C. M. McCullough, M. E. Nieland, J. A. Winger, C. B. Howard, S. Renwick, and D. K. Matheis, Phys. Rev. C 33, 1655 (1986).

${ }^{8}$ G. Rudstam, Z. Naturforschung 21a, 1027 (1966).

${ }^{9}$ C. J. Waddington, Astrophys. Space Sci. 5, 3 (1969).

${ }^{10}$ W. R. Webber (1985) Proceedings of the Workshop on Cosmic
Ray and High Energy Gamma Ray Experiments for the Space Station Era, edited by W. V. Jones and J. P. Wefel (Louisiana State University, Baton Rouge, 1984), p. 283, and private communication.

${ }^{11}$ S. B. Kaufman and E. P. Steinberg, Phys. Rev. C 22, 167 (1980).

${ }^{12}$ W. R. Binns, N. R. Brewster, D. J. Fixsen, T. L. Garrard, M. H. Israel, J. Klarmann, B. J. Newport, E. C. Stone, and C. J. Waddington, Astrophys. J. 297, 111 (1985).

${ }^{13}$ N. R. Brewster, P. S. Freier, and C. J. Waddington, Astrophys. J. 294, 419 (1985).

${ }^{14}$ L. A. Vaishene, L. N. Andronenko, G. G. Kovshevny, A. A. Kotov, and W. Neubert, Z. Phys. A 302, 143 (1981).

${ }^{15}$ C. J. Waddington and P. S. Freier, Phys. Rev. C 31, 888 (1985). 